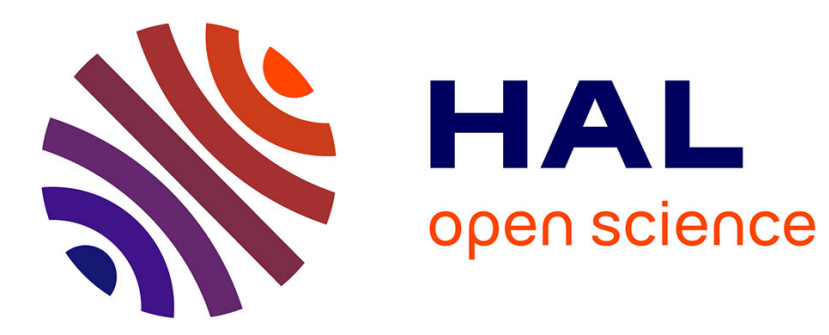

\title{
Influence of the Outer Surface Layers of Crystals on the X-Ray Diffraction Intensity of Basal Reflections
}

\author{
Boris.A. Sakharov, Alain Plançon, Bruno Lanson, Victor.A. Drits
}

\section{To cite this version:}

Boris.A. Sakharov, Alain Plançon, Bruno Lanson, Victor.A. Drits. Influence of the Outer Surface Layers of Crystals on the X-Ray Diffraction Intensity of Basal Reflections. Clays and Clay Minerals, 2004, 52 (6), pp.680-692. 10.1346/ccmn.2004.0520602 . hal-00193925

\section{HAL Id: hal-00193925 \\ https://hal.science/hal-00193925}

Submitted on 5 Dec 2007

HAL is a multi-disciplinary open access archive for the deposit and dissemination of scientific research documents, whether they are published or not. The documents may come from teaching and research institutions in France or abroad, or from public or private research centers.
L'archive ouverte pluridisciplinaire HAL, est destinée au dépôt et à la diffusion de documents scientifiques de niveau recherche, publiés ou non, émanant des établissements d'enseignement et de recherche français ou étrangers, des laboratoires publics ou privés. 


\section{Influence of the Outer Surface Layers of Crystals on the X-Ray Diffraction Intensity of Basal Reflections}

Boris A. Sakharov ${ }^{1}$

Alain Plançon ${ }^{2}$

Bruno Lanson ${ }^{3, *}$

Victor A. Drits ${ }^{1}$

1 - Geological Institute, Russian Academy of Sciences, 7 Pyzhevsky street, 119017 Moscow, Russia.

2 - Crystallography Laboratory, ISTO, University of Orléans - CNRS, 45067 Orléans Cedex 2, France.

3 - Environmental Geochemistry Group, LGIT - Maison des GéoSciences, CNRS University of Grenoble, 38041 Grenoble Cedex 9, France.

* Author to whom correspondence should be addressed.

e-mail: bruno.lanson@obs.ujf-grenoble.fr 


\begin{abstract}
This study presents a mathematical formalism describing diffraction effects from periodic and mixed-layer minerals in which the outer surface layers of crystals differ from layers forming the core of the crystals. XRD patterns calculated for structure models of chlorite and irregular chlorite-smectites terminated on both sides of the crystals by either brucite-like or 2:1 layers show the strong influence that different outer surface layers make on the distribution of basal reflection intensities. Simulation of the experimental XRD patterns from two chlorite samples having different Fe-content shows that in these two samples the chlorite crystals were terminated by brucite-like layers on both sides. In contrast, crystals in a corrensite sample were terminated by water molecules and exchangeable cations. The nature of diffraction effects due to outer surface layers is discussed.
\end{abstract}

\title{
INTRODUCTION
}

Mixed-layer structures (MLSs) are remarkable examples of one-dimensional orderdisorder commonly observed in lamellar crystals. They are composed of layers with different stacking sequences and compositions that alternate in variable proportions and with different distributions. Interstratification effects have been found in structures of various natural and synthetic compounds: layer silicates, phyllomanganates, hydrotalcites and synthetic layered double hydroxides, sulfides, high-temperature superconductors, intercalated graphites, and other lamellar compounds. In natural environments, interlayering is especially widespread among clay minerals (phyllosilicates) which differ in the type of interstratified layers and in their stacking sequences. Two categories may be singled out depending on the distribution of interstratified layer types: first, regular structures in which different layer types alternate periodically along the $c^{*}$ axis and, second, irregular MLSs in which different layer types may either alternate at random or tend to some sort of ordering or segregation. 
Conventional X-ray diffraction (XRD) methods are unsuitable for the structural study of irregular MLSs because of their non-periodic structures, and indirect methods based on the simulation of XRD patterns for different MLS models have been developed. In particular, a matrix formalism has been developed to describe the intensity diffracted by a set of crystals containing different layer types both for basal and hk $\ell$ reflections (Kakinoki and Komura, 1952, 1954a, b, 1965; Drits and Sakharov, 1976; Plançon and Tchoubar, 1976; Plançon, 1981, 2002; Sakharov et al., 1982a, b). In these works it has been systematically assumed that the layers constituting the outer surfaces of the crystals are identical to those in the core of the crystals.

Another approach for calculation of XRD patterns from mixed-layer clay minerals was developed by Reynolds $(1967,1980)$. It is based on the direct summation of the contributions to diffracted intensity coming from waves scattered by all possible layer subsequences existing in the mixed-layer crystals. In the derived algorithm it was assumed that mixed-layer illitesmectites and chlorite-smectites always end on 2:1 layers. Thus, neither formalism can account for the possibility that in natural environments the structure and composition of surface layers of crystals may differ from those of "core" layers. However, according to high-resolution transmission electron microscopy (HRTEM) illite crystals consisting of 2:1 layers may terminate on a 1:1 kaolinite layer (Tsipursky et al., 1992) whereas kaolinite crystals may have pyrophyllite or smectite 2:1 layers as surface terminations (Ma and Eggleton, 1999).

This article proposes a mathematical formalism to simulate XRD patterns from periodic and irregular two-component mixed-layer crystals having any kind of outer surface layers (OSLs). Two useful applications may be related to this formalism. First, it allows estimating the effect of OSLs on diffracted intensity and taking it into account during simulation of the experimental XRD patterns; second, determination of the nature of the microcrystal outer surfaces is essential to the study of their surface properties. 


\section{THEORY}

Amplitude and intensity diffracted by a crystal consisting of $N$ layers

The amplitude diffracted by a layer can be expressed as the product of the layer structure factor $F_{i}$, and of the shape factor $D$. Therefore, the amplitude diffracted by a crystal is:

$$
\mathrm{A}(\overrightarrow{\mathrm{s}})=\sum_{\mathrm{j}=1}^{\mathrm{N}} \mathrm{F}_{\mathrm{j}}(\overrightarrow{\mathrm{s}}) \exp \left(-2 \pi \mathrm{i} \overrightarrow{\mathrm{s}} \overrightarrow{\mathrm{r}}_{\mathrm{oj}}\right) \mathrm{D}(\overrightarrow{\mathrm{s}})
$$

where $\vec{s}$ is the diffusion vector. $\vec{r}_{o j}$ defines the positions of the $\mathrm{j}^{\text {th }}$ layer relative to an arbitrary origin, and $\mathrm{N}$ is the number of layers in the crystal. Note that all layers have the same $\mathrm{D}(\overrightarrow{\mathrm{s}})$ and this term will be omitted in the following developments. In this case, the intensity diffracted by a crystal is:

$$
\mathrm{I}(\overrightarrow{\mathrm{s}})=\mathrm{A}(\overrightarrow{\mathrm{s}}) \mathrm{A}^{*}(\overrightarrow{\mathrm{s}})=\sum_{\mathrm{j}=1}^{\mathrm{N}} \sum_{\mathrm{k}=1}^{\mathrm{N}} \mathrm{F}_{\mathrm{j}}(\overrightarrow{\mathrm{s}}) \mathrm{F}_{\mathrm{k}}^{*}(\overrightarrow{\mathrm{s}}) \exp \left(-2 \pi \mathrm{i} \overrightarrow{\mathrm{s}}\left(\mathrm{r}_{\mathrm{oj}}-\mathrm{r}_{\mathrm{ok}}\right)\right)
$$

where $F_{k}^{*}(\vec{s})$ is the complex amplitude conjugate of $F_{k}(\vec{s})$.

This double summation can be transformed as:

$$
\begin{gathered}
\sum_{j=1}^{N} F_{j}(\vec{s}) F_{j}^{*}(\vec{s})+\sum_{j=1}^{N-1}\left[F_{j}(\vec{s}) F_{j+1}^{*}(\vec{s}) \exp \left(-2 \pi i \vec{s} \vec{r}_{j, j+1}\right)+F_{j}^{*}(\vec{s}) F_{j+1}(\vec{s}) \exp \left(2 \pi i \vec{s} \vec{r}_{j, j+1}\right)\right]+ \\
\sum_{j=1}^{N-2}\left[F_{j}(\vec{s}) F_{j+2}^{*}(\vec{s}) \exp \left(-2 \pi i \vec{s} \vec{r}_{j, j+2}\right)+F_{j}^{*}(\vec{s}) F_{j+2}(\vec{s}) \exp \left(2 \pi i \vec{s} \vec{r}_{j, j+2}\right)\right]+\ldots \ldots+ \\
{\left[F_{1}(\vec{s}) F_{N}^{*}(\vec{s}) \exp \left(-2 \pi i \vec{s} \vec{r}_{1, N}\right)+F_{1}^{*}(\vec{s}) F_{N}(\vec{s}) \exp \left(2 \pi i \vec{s} \vec{r}_{1, N}\right)\right]}
\end{gathered}
$$

At a fixed $\mathrm{j}$ value, the two terms in each bracket are conjugates, and equation (3) becomes:

$$
\sum_{j=1}^{N} F_{j}(\vec{s}) F_{j}^{*}(\vec{s})+2 \operatorname{Re} \sum_{n=1}^{N-1 N-n} \sum_{j=1}^{N} F_{j}(\vec{s}) F_{j+n}^{*}(\vec{s}) \exp \left(-2 \pi i \vec{s} \vec{r}_{j, j+n}\right)
$$

where Re is the real part of the terms in the double summation.

As can be seen in equation (3) the development of the double summation leads to $\mathrm{N}$ terms corresponding to structure factors of individual layers in the crystal and to $2(\mathrm{~N}-\mathrm{n})$ terms 
corresponding to the product of structure factors of the first and final layers in all possible $(n+1)$ layer subsequences. Each product is multiplied by the corresponding phase term which takes into account the phase difference of waves scattered by the terminal layers in each of the $(\mathrm{n}+1)$ layer subsequences. As follows from equation (3), $\mathrm{n}$ varies from 0 to $\mathrm{N}-1$ and equation

(3) thus consists of:

- $\mathrm{N}$ terms for $\mathrm{n}=0(\mathrm{j}=1,2, \ldots, \mathrm{N})$

- 2(N-1) terms for $n=1(j=1,2, \ldots, N)$

- and $2(N-n)$ terms for a given $n^{\text {th }}$ neighbour of a layer $(2 \leq n \leq N-1)$.

The intensity diffracted by a set of $\mathrm{M}$ crystals each containing $\mathrm{N}$ layers can be written:

$$
\operatorname{Int}(\overrightarrow{\mathrm{s}})=[\mathrm{I}(\overrightarrow{\mathrm{s}})]^{1}+[\mathrm{I}(\overrightarrow{\mathrm{s}})]^{2}+\ldots+[\mathrm{I}(\overrightarrow{\mathrm{s}})]^{\mathrm{M}}=\sum_{\mathrm{m}=1}^{\mathrm{M}}[\mathrm{I}(\overrightarrow{\mathrm{s}})]^{\mathrm{m}}
$$

The calculation of $\operatorname{Int}(\overrightarrow{\mathrm{s}})$ requires knowing the nature of the layers at each level of the crystal and for all crystals. Obviously this is never possible and intensity calculations should be carried out for models characterized by average structural parameters. As in previous works (Kakinoki and Komura, 1965; Drits and Sakharov, 1976; Plançon and Tchoubar, 1976; Plançon, 1981; Sakharov et al., 1982a, b) it is assumed in the present study that the layer type distributions in powdered mixed-layer samples obey Markovian statistics.

An important parameter in this model is the short-range order factor R defined as the number of preceding layers that influence the occurrence probability for a final layer of a given type. If two layer types, $A$ and $B$, alternate with $R=1$, then six probability parameters $\left(\mathrm{W}_{\mathrm{A}}, \mathrm{W}_{\mathrm{B}}\right.$, $\left.\mathrm{P}_{\mathrm{AA}}, \mathrm{P}_{\mathrm{AB}}, \mathrm{P}_{\mathrm{BA}}, \mathrm{P}_{\mathrm{BB}}\right)$ are necessary to describe the layer stacking sequence. $\mathrm{W}_{\mathrm{j}}$ is the occurrence probability for layers of type $\mathrm{j}, \mathrm{P}_{\mathrm{jk}}$ is the conditional probability of a layer type $\mathrm{k}$ following a layer type $\mathrm{j}(\mathrm{j}, \mathrm{k}=\mathrm{A}, \mathrm{B})$. Using this set of probability parameters the occurrence probability for any layer subsequence can be easily calculated for a given $\mathrm{R}$ value. For example, when $\mathrm{R}=1$ :

$$
\mathrm{W}_{\mathrm{AA}}=\mathrm{W}_{\mathrm{A}} \mathrm{P}_{\mathrm{AA}}, \mathrm{W}_{\mathrm{AB}}=\mathrm{W}_{\mathrm{A}} \mathrm{P}_{\mathrm{AB}}, \mathrm{W}_{\mathrm{ABA}}=\mathrm{W}_{\mathrm{A}} \mathrm{P}_{\mathrm{AB}} \mathrm{P}_{\mathrm{BA}} \text {, etc }
$$

In addition: 


$$
\sum_{\mathrm{j}} \mathrm{W}_{\mathrm{j}}=1, \sum_{\mathrm{k}} \mathrm{P}_{\mathrm{jk}}=1 \text {, and } \sum_{\mathrm{j}} \mathrm{W}_{\mathrm{j}} \mathrm{P}_{\mathrm{jk}}=\mathrm{W}_{\mathrm{k}}(\mathrm{j}, \mathrm{k}=\mathrm{A}, \mathrm{B})
$$

To further develop equation (3) let us consider at first the term with $n=1$, then $n=2$, then deduce a general term for $1 \leq \mathrm{n} \leq \mathrm{N}-2$, and finally calculate the two particular cases with $\mathrm{n}=0$ and $\mathrm{n}=\mathrm{N}-1$.

Contribution of the $1^{\text {st }}$ neighbor term, $T_{1}$, to the diffracted intensity. Let us assume that the "core" of the crystals (i.e. apart from the OSLs) consist of A and B layers and that $\mathrm{N}_{\mathrm{c}}=\mathrm{N}-2$ is the number of layers in the "core" of the crystal. A' and B' represent the type of layers on one of the outer surfaces $(j=1)$ and $A^{\prime \prime}$ and $B "$ the layer types of the other outer surface $(j=N)$. The contribution of all layer pairs in the crystal is then:

$$
\mathrm{T}_{1}=2 \operatorname{Re} \sum_{\mathrm{m}=1}^{\mathrm{M}} \sum_{\mathrm{j}=1}^{\mathrm{N}-1}\left[\mathrm{~F}_{\mathrm{j}}(\overrightarrow{\mathrm{s}}) \mathrm{F}_{\mathrm{j}+1}^{*}(\overrightarrow{\mathrm{s}}) \exp \left(-2 \pi \mathrm{i} \overrightarrow{\mathrm{s}} \overrightarrow{\mathrm{r}}_{\mathrm{j}, \mathrm{j}+1}\right)\right]^{\mathrm{m}}
$$

which is the sum of the contributions from all layer pairs in the cores of the crystals as well as from layer pairs formed by each OSL with the other layers of the crystals. Using the Markovian statistics the contribution of the "core" layer pairs can be represented as:

$$
2 \mathrm{MRe}\left(\mathrm{N}_{\mathrm{C}}-1\right)\left(\mathrm{W}_{\mathrm{AA}} \mathrm{F}_{\mathrm{A}} \mathrm{F}_{\mathrm{A}}^{*} \varphi_{\mathrm{AA}}+\mathrm{W}_{\mathrm{AB}} \mathrm{F}_{\mathrm{A}} \mathrm{F}_{\mathrm{B}}^{*} \varphi_{\mathrm{AB}}+\mathrm{W}_{\mathrm{BA}} \mathrm{F}_{\mathrm{B}} \mathrm{F}_{\mathrm{A}}^{*} \varphi_{\mathrm{BA}}+\mathrm{W}_{\mathrm{BB}} \mathrm{F}_{\mathrm{B}} \mathrm{F}_{\mathrm{B}}^{*} \varphi_{\mathrm{BB}}\right)
$$

where $\varphi_{\mathrm{jk}}=\exp \left(-2 \pi \mathrm{i} \overrightarrow{\mathrm{s}}\left(\overrightarrow{\mathrm{r}}_{\mathrm{oj}}-\overrightarrow{\mathrm{r}}_{\mathrm{ok}}\right)\right)=\exp \left(-2 \pi \mathrm{i} \overrightarrow{\mathrm{s}} \overrightarrow{\mathrm{t}}_{\mathrm{jk}}\right)$ and $\overrightarrow{\mathrm{t}}_{\mathrm{jk}}$ are the translations relating $\mathrm{j}-$ and $\mathrm{k}-$ type layers $(\mathrm{j}, \mathrm{k}=\mathrm{A}, \mathrm{B})$.

To obtain the total term $T_{1}$, contributions of the two OSLs should be added:

$$
\begin{aligned}
\mathrm{T}_{1} & =2 \mathrm{M} \operatorname{Re}\left[\mathrm{W}_{\mathrm{A}^{\prime} \mathrm{A}} \mathrm{F}_{\mathrm{A}^{\prime}} \mathrm{F}_{\mathrm{A}}^{*} \varphi_{\mathrm{A}^{\prime} \mathrm{A}}+\mathrm{W}_{\mathrm{A}^{\prime} \mathrm{B}} \mathrm{F}_{\mathrm{A}^{\prime}} \mathrm{F}_{\mathrm{B}}^{*} \varphi_{\mathrm{A}^{\prime} \mathrm{B}}+\mathrm{W}_{\mathrm{B}^{\prime} \mathrm{A}} \mathrm{F}_{\mathrm{B}^{\prime}} \mathrm{F}_{\mathrm{A}}^{*} \varphi_{\mathrm{B}^{\prime} \mathrm{A}}+\mathrm{W}_{\mathrm{B}^{\prime} \mathrm{B}} \mathrm{F}_{\mathrm{B}^{\prime}} \mathrm{F}_{\mathrm{B}}^{*} \varphi_{\mathrm{B}^{\prime} \mathrm{B}}\right. \\
& +\left(\mathrm{N}_{\mathrm{C}}-1\right)\left(\mathrm{W}_{\mathrm{AA}} \mathrm{F}_{\mathrm{A}} \mathrm{F}_{\mathrm{A}}^{*} \varphi_{\mathrm{AA}}+\mathrm{W}_{\mathrm{AB}} \mathrm{F}_{\mathrm{A}} \mathrm{F}_{\mathrm{B}}^{*} \varphi_{\mathrm{AB}}+\mathrm{W}_{\mathrm{BA}} \mathrm{F}_{\mathrm{B}} \mathrm{F}_{\mathrm{A}}^{*} \varphi_{\mathrm{BA}}+\mathrm{W}_{\mathrm{BB}} \mathrm{F}_{\mathrm{B}} \mathrm{F}_{\mathrm{B}}^{*} \varphi_{\mathrm{BB}}\right) \\
& \left.+\mathrm{W}_{\mathrm{AA}^{\prime \prime}} \mathrm{F}_{\mathrm{A}} \mathrm{F}_{\mathrm{A}^{\prime \prime}}^{*} \varphi_{\mathrm{AA}^{\prime \prime}}+\mathrm{W}_{\mathrm{AB}^{\prime \prime}} \mathrm{F}_{\mathrm{A}} \mathrm{F}_{\mathrm{B}^{\prime \prime}}^{*} \varphi_{\mathrm{AB}^{\prime \prime}}+\mathrm{W}_{\mathrm{BA}^{\prime \prime}} \mathrm{F}_{\mathrm{B}} \mathrm{F}_{\mathrm{A}^{\prime \prime}}^{*} \varphi_{\mathrm{BA}^{\prime \prime}}+\mathrm{W}_{\mathrm{BB}^{\prime}} \mathrm{F}_{\mathrm{B}} \mathrm{F}_{\mathrm{B}^{\prime \prime}}^{*} \varphi_{\mathrm{BB}^{\prime \prime}}\right]
\end{aligned}
$$

In addition, occurrence probability and phase terms of the A'A layer pairs are identical to that of AA layer pairs (the A' layer is merely a "scratched" or "covered" A layer) so that 
$\mathrm{W}_{\mathrm{A}^{\prime} \mathrm{A}}=\mathrm{W}_{\mathrm{AA}}$. The same is true for $\mathrm{A}^{\prime} \mathrm{B}$ and $\mathrm{AB}, \mathrm{B}^{\prime} \mathrm{A}$ and $\mathrm{BA}, \mathrm{B}^{\prime} \mathrm{B}$ and $\mathrm{BB}$, for $\mathrm{AA}$ " and $\mathrm{AA}$, $\mathrm{AB}^{\prime \prime}$ and $\mathrm{AB}, \mathrm{BA}^{\prime \prime}$ and $\mathrm{BA}$, and for $\mathrm{BB} "$ and $\mathrm{BB}$. Then,

$$
\begin{gathered}
\mathrm{T}_{1}=2 \mathrm{MRe}\left[\mathrm{W}_{\mathrm{AA}} \mathrm{F}_{\mathrm{A}^{\prime}} \mathrm{F}_{\mathrm{A}}^{*} \varphi_{\mathrm{AA}}+\mathrm{W}_{\mathrm{AB}} \mathrm{F}_{\mathrm{A}^{\prime}} \mathrm{F}_{\mathrm{B}}^{*} \varphi_{\mathrm{AB}}+\mathrm{W}_{\mathrm{BA}} \mathrm{F}_{\mathrm{B}} \mathrm{F}_{\mathrm{A}}^{*} \varphi_{\mathrm{BA}}+\mathrm{W}_{\mathrm{BB}} \mathrm{F}_{\mathrm{B}} \mathrm{F}_{\mathrm{B}}^{*} \varphi_{\mathrm{BB}}\right. \\
+\left(\mathrm{N}_{\mathrm{C}}-1\right)\left(\mathrm{W}_{\mathrm{AA}} \mathrm{F}_{\mathrm{A}} \mathrm{F}_{\mathrm{A}}^{*} \varphi_{\mathrm{AA}}+\mathrm{W}_{\mathrm{AB}} \mathrm{F}_{\mathrm{A}} \mathrm{F}_{\mathrm{B}}^{*} \varphi_{\mathrm{AB}}+\mathrm{W}_{\mathrm{BA}} \mathrm{F}_{\mathrm{B}} \mathrm{F}_{\mathrm{A}}^{*} \varphi_{\mathrm{BA}}+\mathrm{W}_{\mathrm{BB}} \mathrm{F}_{\mathrm{B}} \mathrm{F}_{\mathrm{B}}^{*} \varphi_{\mathrm{BB}}\right) \\
\left.+\mathrm{W}_{\mathrm{AA}} \mathrm{F}_{\mathrm{A}} \mathrm{F}_{\mathrm{A}^{\prime \prime}}^{*} \varphi_{\mathrm{AA}}+\mathrm{W}_{\mathrm{AB}} \mathrm{F}_{\mathrm{A}} \mathrm{F}_{\mathrm{B}}^{*} \varphi_{\mathrm{AB}}+\mathrm{W}_{\mathrm{BA}} \mathrm{F}_{\mathrm{B}} \mathrm{F}_{\mathrm{A}}^{*} \varphi_{\mathrm{BA}}+\mathrm{W}_{\mathrm{BB}} \mathrm{F}_{\mathrm{B}} \mathrm{F}_{\mathrm{B}}^{*} \varphi_{\mathrm{BB}}\right]
\end{gathered}
$$

Thus, the term $\mathrm{T}_{1}$ can be presented using the matrix formalism proposed by different authors (Drits and Sakharov, 1976; Plançon and Tchoubar, 1976; Plançon, 1981, Sakharov et al., 1982a) for crystals without specific outer surfaces.

$$
\mathrm{T}_{1}=2 \mathrm{M} \operatorname{Spur}\left(\operatorname{Re}\left(\left(\Phi_{1 \mathrm{C}}+\left(\mathrm{N}_{\mathrm{C}}-1\right) \Phi_{\mathrm{CC}}+\Phi_{\mathrm{CN}}\right) \mathrm{WQ}^{1}\right)\right)
$$

where matrices of the second rank $\Phi_{1 \mathrm{C}}, \Phi_{\mathrm{CC}}, \Phi_{\mathrm{CN}}, \mathrm{W}$ and $\mathrm{Q}$ are presented as:

$$
\begin{gathered}
\Phi_{1 \mathrm{C}}=\left(\begin{array}{cc}
\mathrm{F}_{\mathrm{A}} \cdot \mathrm{F}_{\mathrm{A}}^{*} & \mathrm{~F}_{\mathrm{B}} \cdot \mathrm{F}_{\mathrm{A}}^{*} \\
\mathrm{~F}_{\mathrm{A}} \cdot \mathrm{F}_{\mathrm{B}}^{*} & \mathrm{~F}_{\mathrm{B}} \cdot \mathrm{F}_{\mathrm{B}}^{*}
\end{array}\right) \Phi_{\mathrm{CC}}=\left(\begin{array}{ll}
\mathrm{F}_{\mathrm{A}} \mathrm{F}_{\mathrm{A}}^{*} & \mathrm{~F}_{\mathrm{B}} \mathrm{F}_{\mathrm{A}}^{*} \\
\mathrm{~F}_{\mathrm{A}} \mathrm{F}_{\mathrm{B}}^{*} & \mathrm{~F}_{\mathrm{B}} \mathrm{F}_{\mathrm{B}}^{*}
\end{array}\right) \quad \Phi_{\mathrm{CN}}=\left(\begin{array}{ll}
\mathrm{F}_{\mathrm{A}} \mathrm{F}_{\mathrm{A}}^{*} & \mathrm{~F}_{\mathrm{B}} \mathrm{F}_{\mathrm{A}^{\prime \prime}}^{*} \\
\mathrm{~F}_{\mathrm{A}} \mathrm{F}_{\mathrm{B}}^{*} & \mathrm{~F}_{\mathrm{B}} \mathrm{F}_{\mathrm{B}^{\prime \prime}}^{*}
\end{array}\right) \\
\mathrm{W}=\left(\begin{array}{cc}
\mathrm{W}_{\mathrm{A}} & 0 \\
0 & \mathrm{~W}_{\mathrm{B}}
\end{array}\right) \quad \mathrm{Q}=\left(\begin{array}{ll}
\mathrm{p}_{\mathrm{AA}} \varphi_{\mathrm{AA}} & \mathrm{p}_{\mathrm{AB}} \varphi_{\mathrm{AB}} \\
\mathrm{p}_{\mathrm{BA}} \varphi_{\mathrm{BA}} & \mathrm{p}_{\mathrm{BB}} \varphi_{\mathrm{BB}}
\end{array}\right)
\end{gathered}
$$

where the subscript $\mathrm{C}$ refers to layers in the "core" of the crystals, 1 refers to the first (i.e. one of the outer surfaces) and $\mathrm{N}$ to the $\mathrm{N}^{\text {th }}$ layer (i.e the other outer surface).

Contribution of the $2^{\text {nd }}$ neighbor terms, $T_{2}$, to the diffracted intensity. The term $\mathrm{T}_{2}$ which includes the contributions of all layer triplets in the crystals is equal to:

$$
\begin{aligned}
& \mathrm{T}_{2}=2 \operatorname{Re} \sum_{\mathrm{m}=1}^{\mathrm{M}} \sum_{\mathrm{j}=1}^{\mathrm{N}-2}\left[\mathrm{~F}_{\mathrm{j}}(\overrightarrow{\mathrm{s}}) \mathrm{F}_{\mathrm{j}+2}^{*}(\overrightarrow{\mathrm{s}}) \exp -2 \pi \mathrm{i} \overrightarrow{\mathrm{s}} \overrightarrow{\mathrm{r}}_{\mathrm{j}, \mathrm{j}+2}\right]^{\mathrm{m}} \text { or } \\
& \mathrm{T}_{2}=2 \operatorname{Re}\left\{\left[\mathrm{W}_{\mathrm{A}^{\prime} \mathrm{AA}} \mathrm{F}_{\mathrm{A}^{\prime}} \mathrm{F}_{\mathrm{A}}^{*} \varphi_{\mathrm{A}^{\prime} \mathrm{AA}}+\mathrm{W}_{\mathrm{A}^{\prime} \mathrm{BA}} \mathrm{F}_{\mathrm{A}^{\prime}} \mathrm{F}_{\mathrm{A}}^{*} \varphi_{\mathrm{A}^{\prime} \mathrm{BA}}+\mathrm{W}_{\mathrm{A}^{\prime} \mathrm{AB}} \mathrm{F}_{\mathrm{A}^{\prime}} \mathrm{F}_{\mathrm{B}}^{*} \varphi_{\mathrm{A}^{\prime} \mathrm{AB}}+\mathrm{W}_{\mathrm{A}^{\prime} \mathrm{BB}} \mathrm{F}_{\mathrm{A}^{\prime}} \mathrm{F}_{\mathrm{B}}^{*} \varphi_{\mathrm{A}^{\prime} \mathrm{BB}}\right.\right. \\
& \left.+\mathrm{W}_{\mathrm{B}^{\prime} \mathrm{AA}} \mathrm{F}_{\mathrm{B}^{\prime}} \mathrm{F}_{\mathrm{A}}^{*} \varphi_{\mathrm{B}^{\prime} \mathrm{AA}}+\mathrm{W}_{\mathrm{B}^{\prime} \mathrm{BA}} \mathrm{F}_{\mathrm{B}^{\prime}} \mathrm{F}_{\mathrm{A}}^{*} \varphi_{\mathrm{B}^{\prime} \mathrm{BA}}+\mathrm{W}_{\mathrm{B}^{\prime} \mathrm{AB}} \mathrm{F}_{\mathrm{B}^{\prime}} \mathrm{F}_{\mathrm{B}}^{*} \varphi_{\mathrm{B}^{\prime} \mathrm{AB}}+\mathrm{W}_{\mathrm{B}^{\prime} \mathrm{BB}} \mathrm{F}_{\mathrm{B}^{\prime}} \mathrm{F}_{\mathrm{B}}^{*} \varphi_{\mathrm{B}^{\prime} \mathrm{BB}}\right] \\
& +\left(\mathrm{N}_{\mathrm{c}}-2\right)\left[\mathrm{W}_{\mathrm{AAA}} \mathrm{F}_{\mathrm{A}} \mathrm{F}_{\mathrm{A}}^{*} \varphi_{\mathrm{AAA}}+\mathrm{W}_{\mathrm{ABA}} \mathrm{F}_{\mathrm{A}} \mathrm{F}_{\mathrm{A}}^{*} \varphi_{\mathrm{ABA}}+\mathrm{W}_{\mathrm{AAB}} \mathrm{F}_{\mathrm{A}} \mathrm{F}_{\mathrm{B}}^{*} \varphi_{\mathrm{AAB}}+\mathrm{W}_{\mathrm{ABB}} \mathrm{F}_{\mathrm{A}} \mathrm{F}_{\mathrm{B}}^{*} \varphi_{\mathrm{ABB}}\right. \\
& \left.+\mathrm{W}_{\mathrm{BAA}} \mathrm{F}_{\mathrm{B}} \mathrm{F}_{\mathrm{A}}^{*} \varphi_{\mathrm{BAA}}+\mathrm{W}_{\mathrm{BBA}} \mathrm{F}_{\mathrm{B}} \mathrm{F}_{\mathrm{A}}^{*} \varphi_{\mathrm{BBA}}+\mathrm{W}_{\mathrm{BAB}} \mathrm{F}_{\mathrm{B}} \mathrm{F}_{\mathrm{B}}^{*} \varphi_{\mathrm{BAB}}+\mathrm{W}_{\mathrm{BBB}} \mathrm{F}_{\mathrm{B}} \mathrm{F}_{\mathrm{B}}^{*} \varphi_{\mathrm{BBB}}\right]
\end{aligned}
$$




$$
\begin{gathered}
+\left[\mathrm{W}_{\mathrm{AAA}^{\prime \prime}} \mathrm{F}_{\mathrm{A}} \mathrm{F}_{\mathrm{A}^{\prime \prime}}^{*} \varphi_{\mathrm{AAA}^{\prime \prime}}+\mathrm{W}_{\mathrm{ABA}^{\prime \prime}} \mathrm{F}_{\mathrm{A}} \mathrm{F}_{\mathrm{A}^{\prime \prime}}^{*} \varphi_{\mathrm{ABA}^{\prime \prime}}+\mathrm{W}_{\mathrm{AAB}^{\prime \prime}} \mathrm{F}_{\mathrm{A}} \mathrm{F}_{\mathrm{B}^{\prime \prime}}^{*} \varphi_{\mathrm{AAB}^{\prime \prime}}+\mathrm{W}_{\mathrm{ABB}^{\prime \prime}} \mathrm{F}_{\mathrm{A}} \mathrm{F}_{\mathrm{B}^{\prime \prime}}^{*} \varphi_{\mathrm{ABB}^{\prime \prime}}\right. \\
\left.\left.+\mathrm{W}_{\mathrm{BAA}^{\prime \prime}} \mathrm{F}_{\mathrm{B}} \mathrm{F}_{\mathrm{A}^{\prime \prime}}^{*} \varphi_{\mathrm{BAA}^{\prime \prime}}+\mathrm{W}_{\mathrm{BBA}^{\prime}} \mathrm{F}_{\mathrm{B}} \mathrm{F}_{\mathrm{A}^{\prime \prime}}^{*} \varphi_{\mathrm{BBA}^{\prime \prime}}+\mathrm{W}_{\mathrm{BAB}^{\prime \prime}} \mathrm{F}_{\mathrm{B}} \mathrm{F}_{\mathrm{B}^{\prime \prime}}^{*} \varphi_{\mathrm{BAB}^{\prime \prime}}+\mathrm{W}_{\mathrm{BBB}^{\prime}} \mathrm{F}_{\mathrm{B}} \mathrm{F}_{\mathrm{B}^{\prime \prime}}^{*} \varphi_{\mathrm{BBB}^{\prime \prime}}\right]\right\}
\end{gathered}
$$

As for $T_{1}$, the term $T_{2}$ can also be expressed using the matrix formalism:

$$
\mathrm{T}_{2}=2 \mathrm{M} \operatorname{Spur}\left(\operatorname{Re}\left(\left(\Phi_{1 \mathrm{C}}+\left(\mathrm{N}_{\mathrm{C}}-2\right) \Phi_{\mathrm{CC}}+\Phi_{\mathrm{CN}}\right) \mathrm{WQ}^{2}\right)\right)
$$

Contribution of the $n^{\text {th }}$ neighbor term, $T_{n}(1 \leq n \leq N-2)$, to the diffracted intensity. This term can be obtained recursively and may be expressed as:

$$
\mathrm{T}_{\mathrm{n}}=2 \mathrm{M} \operatorname{Spur}\left(\operatorname{Re}\left(\left(\Phi_{1 \mathrm{C}}+\left(\mathrm{N}_{\mathrm{C}}-\mathrm{n}\right) \Phi_{\mathrm{CC}}+\Phi_{\mathrm{CN}}\right) \mathrm{WQ}^{\mathrm{n}}\right)\right)
$$

Contribution to the diffraction of the $(N-1)^{\text {th }}$ neighbor term. This term involves only the layers of the two outer surfaces:

$$
\mathrm{T}_{\mathrm{N}-1}=2 \mathrm{M} \operatorname{Spur}\left(\operatorname{Re}\left(\Phi_{1 \mathrm{~N}} \mathrm{WQ}^{\mathrm{N}-1}\right)\right)
$$

with $\Phi_{1 \mathrm{~N}}=\left(\begin{array}{ll}\mathrm{F}_{\mathrm{A}^{\prime}} \mathrm{F}_{\mathrm{A}^{\prime \prime}}^{*} & \mathrm{~F}_{\mathrm{B}^{\prime}} \mathrm{F}_{\mathrm{A}^{\prime \prime}}^{*} \\ \mathrm{~F}_{\mathrm{A}^{\prime}} \mathrm{F}_{\mathrm{B}^{\prime \prime}}^{*} & \mathrm{~F}_{\mathrm{B}^{\prime}} \mathrm{F}_{\mathrm{B}^{\prime \prime}}^{*}\end{array}\right)$.

Contribution of the $0^{\text {th }}$ neighbor term, $T_{0}$, to the diffracted intensity. This term describes the contribution of individual layers without interactions with neighboring layers.

$$
\mathrm{T}_{0}=\mathrm{M} \operatorname{Spur}\left(\operatorname{Re}\left(\left(\Phi_{11}+\mathrm{N}_{\mathrm{C}} \Phi_{\mathrm{CC}}+\Phi_{\mathrm{NN}}\right) \mathrm{W}\right)\right)
$$

with $\Phi_{11}=\left(\begin{array}{cc}\mathrm{F}_{\mathrm{A}^{\prime}} \mathrm{F}_{\mathrm{A}^{\prime}}^{*} & 0 \\ 0 & \mathrm{~F}_{\mathrm{B}^{\prime}} \mathrm{F}_{\mathrm{B}^{\prime}}^{*}\end{array}\right)$, and $\Phi_{\mathrm{NN}}=\left(\begin{array}{cc}\mathrm{F}_{\mathrm{A}^{\prime \prime}} \mathrm{F}_{\mathrm{A}^{\prime \prime}}^{*} & 0 \\ 0 & \mathrm{~F}_{\mathrm{B}^{\prime \prime}} \mathrm{F}_{\mathrm{B}^{\prime \prime}}^{*}\end{array}\right)$.

Grouping all terms together. Summing up all the above $\mathrm{T}_{\mathrm{n}}$ terms, the intensity equation becomes: 
$\operatorname{Int}(\overrightarrow{\mathrm{s}})=\operatorname{MSpur}\left(\operatorname{Re}\left(\left(\phi_{11}+\mathrm{N}_{\mathrm{C}} \phi_{\mathrm{CC}}+\phi_{\mathrm{NN}}\right) \mathrm{W}+2 \sum_{\mathrm{n}=1}^{\mathrm{N}_{\mathrm{C}}}\left(\phi_{1 \mathrm{C}}+\left(\mathrm{N}_{\mathrm{C}}-\mathrm{n}\right) \phi_{\mathrm{CC}}+\phi_{\mathrm{CN}}\right) \mathrm{WQ}^{\mathrm{n}}+2 \phi_{1 \mathrm{~N}} \mathrm{WQ}^{\mathrm{N}-1}\right)\right)$

If the layers of the outer surfaces are the same as those of the core of the crystals (i.e. $\mathrm{A}^{\prime} \equiv \mathrm{A} \equiv \mathrm{A}^{\prime \prime}$ and $\left.\mathrm{B}^{\prime} \equiv \mathrm{B} \equiv \mathrm{B}^{\prime \prime}\right)$, then the intensity equation is simplified because $\Phi_{11}=\Phi_{\mathrm{CC}}=\Phi_{\mathrm{NN}}=$ $\Phi_{1 \mathrm{C}}=\Phi_{\mathrm{CN}}=\Phi_{1 \mathrm{~N}}=\Phi$ and becomes, as described earlier (Drits and Sakharov, 1976; Plançon and Tchoubar, 1976; Plançon, 1981, 2003; Sakharov et al., 1982a):

$$
\operatorname{Int}(\overrightarrow{\mathrm{s}})=\operatorname{MSpur}\left(\operatorname{Re}\left(\phi \mathrm{W}\left(\mathrm{NE}+2 \sum_{n=1}^{\mathrm{N}-1}(\mathrm{~N}-\mathrm{n}) \mathrm{Q}^{\mathrm{n}}\right)\right)\right)
$$

where $\mathrm{E}$ is the unit matrix.

The average intensity diffracted by a crystal consisting of $\mathrm{N}$ layers is thus equal to $\overline{\operatorname{Int}}(\overrightarrow{\mathrm{s}})=\operatorname{Int}(\overrightarrow{\mathrm{s}}) / \mathrm{M}$.

If the range of interaction between layers is greater than 1 the basis of the formalism remains the same, but the rank of the matrices increases as explained by Drits and Sakharov (1976), Plançon (1981), Sakharov et al. (1982a), and Drits and Tchoubar (1990). In particular, for a two-component MLS with $\mathrm{R}=2$ the matrices are:

$$
\begin{aligned}
& \mathrm{W}=\left(\begin{array}{llll}
\mathrm{W}_{\mathrm{AA}} & & & \\
& \mathrm{W}_{\mathrm{AB}} & & \\
& & \mathrm{W}_{\mathrm{BA}} & \\
& & & \mathrm{W}_{\mathrm{BB}}
\end{array}\right), \\
& \mathrm{Q}=\left(\begin{array}{llll}
\mathrm{P}_{\mathrm{AAA}} \varphi_{\mathrm{AA}} & \mathrm{P}_{\mathrm{AAB}} \varphi_{\mathrm{AA}} & & \\
& & \mathrm{P}_{\mathrm{ABA}} \varphi_{\mathrm{AB}} & \mathrm{P}_{\mathrm{ABB}} \varphi_{\mathrm{AB}} \\
\mathrm{P}_{\mathrm{BAA}} \varphi_{\mathrm{BA}} & \mathrm{P}_{\mathrm{BAB}} \varphi_{\mathrm{BA}} & & \\
& & \mathrm{P}_{\mathrm{BBA}} \varphi_{\mathrm{BB}} & \mathrm{P}_{\mathrm{BBB}} \varphi_{\mathrm{BB}}
\end{array}\right), \\
& \Phi_{\mathrm{CC}}=\left(\begin{array}{cccc}
\mathrm{F}_{\mathrm{A}} \mathrm{F}_{\mathrm{A}}^{*} & \mathrm{~F}_{\mathrm{A}} \mathrm{F}_{\mathrm{A}}^{*} & \mathrm{~F}_{\mathrm{B}} \mathrm{F}_{\mathrm{A}}^{*} & \mathrm{~F}_{\mathrm{B}} \mathrm{F}_{\mathrm{A}}^{*} \\
\mathrm{~F}_{\mathrm{A}} \mathrm{F}_{\mathrm{A}}^{*} & \mathrm{~F}_{\mathrm{A}} \mathrm{F}_{\mathrm{A}}^{*} & \mathrm{~F}_{\mathrm{B}} \mathrm{F}_{\mathrm{A}}^{*} & \mathrm{~F}_{\mathrm{B}} \mathrm{F}_{\mathrm{A}}^{*} \\
\mathrm{~F}_{\mathrm{A}} \mathrm{F}_{\mathrm{B}}^{*} & \mathrm{~F}_{\mathrm{A}} \mathrm{F}_{\mathrm{B}}^{*} & \mathrm{~F}_{\mathrm{B}} \mathrm{F}_{\mathrm{B}}^{*} & \mathrm{~F}_{\mathrm{B}} \mathrm{F}_{\mathrm{B}}^{*} \\
\mathrm{~F}_{\mathrm{A}} \mathrm{F}_{\mathrm{B}}^{*} & \mathrm{~F}_{\mathrm{A}} \mathrm{F}_{\mathrm{B}}^{*} & \mathrm{~F}_{\mathrm{B}} \mathrm{F}_{\mathrm{B}}^{*} & \mathrm{~F}_{\mathrm{B}} \mathrm{F}_{\mathrm{B}}^{*}
\end{array}\right)
\end{aligned}
$$




$$
\begin{aligned}
& \Phi_{1 C}=\left(\begin{array}{cccc}
F_{A} \cdot F_{A}^{*} & F_{A} \cdot F_{A}^{*} & F_{B} \cdot F_{A}^{*} & F_{B} \cdot F_{A}^{*} \\
F_{A} \cdot F_{A}^{*} & F_{A} \cdot F_{A}^{*} & F_{B} \cdot F_{A}^{*} & F_{B} \cdot F_{A}^{*} \\
F_{A} \cdot F_{B}^{*} & F_{A} \cdot F_{B}^{*} & F_{B} \cdot F_{B}^{*} & F_{B} \cdot F_{B}^{*} \\
F_{A} \cdot F_{B}^{*} & F_{A} \cdot F_{B}^{*} & F_{B} \cdot F_{B}^{*} & F_{B} \cdot F_{B}^{*}
\end{array}\right), \\
& \Phi_{\mathrm{CN}}=\left(\begin{array}{llll}
\mathrm{F}_{\mathrm{A}} \mathrm{F}_{\mathrm{A}^{\prime \prime}}^{*} & \mathrm{~F}_{\mathrm{A}} \mathrm{F}_{\mathrm{A}^{\prime \prime}}^{*} & \mathrm{~F}_{\mathrm{B}} \mathrm{F}_{\mathrm{A}^{\prime \prime}}^{*} & \mathrm{~F}_{\mathrm{B}} \mathrm{F}_{\mathrm{A}^{\prime \prime}}^{*} \\
\mathrm{~F}_{\mathrm{A}} \mathrm{F}_{\mathrm{A}^{\prime \prime}}^{*} & \mathrm{~F}_{\mathrm{A}} \mathrm{F}_{\mathrm{A}^{\prime \prime}}^{*} & \mathrm{~F}_{\mathrm{B}} \mathrm{F}_{\mathrm{A}^{\prime \prime}}^{*} & \mathrm{~F}_{\mathrm{B}} \mathrm{F}_{\mathrm{A}^{\prime \prime}}^{*} \\
\mathrm{~F}_{\mathrm{A}} \mathrm{F}_{\mathrm{B}^{\prime \prime}}^{*} & \mathrm{~F}_{\mathrm{A}} \mathrm{F}_{\mathrm{B}^{\prime \prime}}^{*} & \mathrm{~F}_{\mathrm{B}} \mathrm{F}_{\mathrm{B}^{\prime \prime}}^{*} & \mathrm{~F}_{\mathrm{B}} \mathrm{F}_{\mathrm{B}^{\prime \prime}}^{*} \\
\mathrm{~F}_{\mathrm{A}} \mathrm{F}_{\mathrm{B}^{\prime \prime}}^{*} & \mathrm{~F}_{\mathrm{A}} \mathrm{F}_{\mathrm{B}^{\prime \prime}}^{*} & \mathrm{~F}_{\mathrm{B}} \mathrm{F}_{\mathrm{B}^{\prime \prime}}^{*} & \mathrm{~F}_{\mathrm{B}} \mathrm{F}_{\mathrm{B}^{\prime \prime}}^{*}
\end{array}\right), \\
& \Phi_{11}=\left(\begin{array}{llll}
\mathrm{F}_{\mathrm{A}^{\prime}} \cdot \mathrm{F}_{\mathrm{A}^{\prime}}^{*} & \mathrm{~F}_{\mathrm{A}^{\prime}} \mathrm{F}_{\mathrm{A}^{\prime}}^{*} & & \\
\mathrm{~F}_{\mathrm{A}^{\prime}} \mathrm{F}_{\mathrm{A}^{\prime}}^{*} & \mathrm{~F}_{\mathrm{A}^{\prime}} \mathrm{F}_{\mathrm{A}^{\prime}}^{*} & & \\
& & \mathrm{~F}_{\mathrm{B}^{\prime}} \mathrm{F}_{\mathrm{B}^{\prime}}^{*} & \mathrm{~F}_{\mathrm{B}^{\prime}} \mathrm{F}_{\mathrm{B}^{\prime}}^{*} \\
& & \mathrm{~F}_{\mathrm{B}^{\prime}} \mathrm{F}_{\mathrm{B}^{\prime}}^{*} & \mathrm{~F}_{\mathrm{B}^{\prime}} \mathrm{F}_{\mathrm{B}^{\prime}}^{*}
\end{array}\right) \text {, and } \\
& \Phi_{\mathrm{NN}}=\left(\begin{array}{llll}
\mathrm{F}_{\mathrm{A}^{*}} \mathrm{~F}_{\mathrm{A}^{\prime \prime}}^{*} & \mathrm{~F}_{\mathrm{A}^{\prime \prime}} \mathrm{F}_{\mathrm{A}^{\prime \prime}}^{*} & & \\
\mathrm{~F}_{\mathrm{A}^{*}} \mathrm{~F}_{\mathrm{A}^{*}}^{*} & \mathrm{~F}_{\mathrm{A}^{\prime \prime}} \mathrm{F}_{\mathrm{A}^{\prime \prime}}^{*} & & \\
& & \mathrm{~F}_{\mathrm{B}^{\prime}} \mathrm{F}_{\mathrm{B}^{*}}^{*} & \mathrm{~F}_{\mathrm{B}^{\prime \prime}} \mathrm{F}_{\mathrm{B}^{\prime \prime}}^{*} \\
& & \mathrm{~F}_{\mathrm{B}^{\prime}} \mathrm{F}_{\mathrm{B}^{*}}^{*} & \mathrm{~F}_{\mathrm{B}^{\prime \prime}} \mathrm{F}_{\mathrm{B}^{\prime \prime}}^{*}
\end{array}\right) .
\end{aligned}
$$

In this case, $\mathrm{P}_{\mathrm{ijk}}$ defines the probability of layer type $\mathrm{k}$ to follow a layer pair $\mathrm{ij}$, and the following relationships $\sum_{\mathrm{j}} \sum_{\mathrm{k}} \mathrm{W}_{\mathrm{jk}}=1, \sum_{\mathrm{l}} \mathrm{P}_{\mathrm{jkl}}=1$, and $\sum_{\mathrm{j}} \mathrm{W}_{\mathrm{jk}} \mathrm{P}_{\mathrm{jkl}}=\mathrm{W}_{\mathrm{k} 1}$ complement equation (6). In terms of the Markovian statistics the probability parameters are interrelated and the number of independent parameters for a given $\mathrm{R}$ value is therefore relatively small. For example, in a structure with $\mathrm{R}=1$ and $\mathrm{W}_{\mathrm{A}}>\mathrm{W}_{\mathrm{B}}$ two independent parameters are required to determine all other probability parameters and thus to describe any layer subsequence. Similarly, for a twocomponent system with $\mathrm{R}=2$ and $\mathrm{W}_{\mathrm{A}}>\mathrm{W}_{\mathrm{B}}$ only four independent parameters are required (Drits and Tchoubar, 1990).

\section{Alternate model}

This model describes the case when the OSL is defined by the type of the preceding or following "core" layer. For example, if we assume that an A-type layer is the final core layer, A'-type and A"-type layers are respectively after and before the A-type core layer and it is then 
possible to define new OSLs. Each of the A'-, B'-, A"- and B"-type layers can be combined with the nearest core layer (A- or B-type) to form new layers: $\mathrm{A}_{u}=\mathrm{A}+\mathrm{A}^{\prime}, \mathrm{B}_{u}=\mathrm{B}+\mathrm{B}^{\prime}, \mathrm{A}_{l}=\mathrm{A}^{\prime \prime}+\mathrm{A}$, and $\mathrm{B}_{l}=\mathrm{B} "+\mathrm{B}$, where $u$ and $l$ denote the upper and lower surface layers of the crystal. This modification makes it possible to keep the junction probabilities $\left(\mathrm{P}_{\mathrm{ij}}\right)$ constant over the whole crystal, which in turn facilitates the calculation using the proposed matrix formalism. New structural amplitudes $\mathrm{F}_{\mathrm{A} u}, \mathrm{~F}_{\mathrm{B} u}, \mathrm{~F}_{\mathrm{Al}}$ and $\mathrm{F}_{\mathrm{B} l}$ are introduced in the matrices $\Phi_{1 \mathrm{C}}, \Phi_{\mathrm{CN}}, \Phi_{\mathrm{NN}}, \Phi_{11}$ and $\Phi_{1 \mathrm{~N}}$ to replace $\mathrm{F}_{\mathrm{A}^{\prime}}, \mathrm{F}_{\mathrm{B}^{\prime}}, \mathrm{F}_{\mathrm{A}^{\prime \prime}}$ and $\mathrm{F}_{\mathrm{B}^{\prime \prime}}$. The intensity equation is the same as (19) but the number of core layers in a crystal is reduced to $\mathrm{N}_{\mathrm{c}}-2$.

Intensity diffracted by a set of crystals having different number of layers

If $\mathrm{p}\left(\mathrm{N}_{\mathrm{i}}\right)$ is the proportion of crystals containing $\mathrm{N}_{\mathrm{i}}$ layers, and $\mathrm{N}_{\min }$ and $\mathrm{N}_{\max }$ are the minimum and the maximum number of layers in the crystals, then $\sum_{N_{j}=N_{\min }}^{N_{\max }} p\left(N_{j}\right)=1 . N_{\min }$ can be chosen as $1\left(p(1)=0\right.$ if there is no isolated layers). The case $\mathrm{N}_{\min }=1$ is a particular case because an isolated layer is not A, A', A', or B, B', B'. An isolated A layer must have one surface like $A^{\prime}$ and its other surface like $A^{\prime \prime}$; it is an $A^{\prime \prime \prime}$ layer with a structure factor $F_{A^{\prime \prime}}$ and the same is true for B. As a consequence, a new matrix must be introduced:

$$
\phi_{11^{\prime}}=\left(\begin{array}{cc}
\mathrm{F}_{\mathrm{A}^{\prime \prime}} \mathrm{F}_{\mathrm{A}^{\prime \prime \prime}}^{*} & 0 \\
0 & \mathrm{~F}_{\mathrm{B}^{\prime \prime}} \mathrm{F}_{\mathrm{B}^{\prime \prime}}^{*}
\end{array}\right) \text {, }
$$

In turn:

$$
\begin{gathered}
\overline{\operatorname{Int}}(\overrightarrow{\mathrm{s}})=\operatorname{Spur}\left(\operatorname { R e } \left(\mathrm{p}(1)\left(\phi_{11}, \mathrm{~W}\right)\right.\right. \\
+\mathrm{p}(2)\left(\left(\phi_{11}+\phi_{\mathrm{NN}}\right) \mathrm{W}+2 \phi_{1 \mathrm{~N}} \mathrm{WQ}\right) \\
+\mathrm{p}(3)\left(\left(\phi_{11}+\phi_{\mathrm{CC}}+\phi_{\mathrm{NN}}\right) \mathrm{W}+2\left(\phi_{1 \mathrm{C}}+\phi_{\mathrm{CN}}\right) \mathrm{WQ}^{1}+2 \phi_{1 \mathrm{~N}} \mathrm{WQ}^{2}\right) \\
+\ldots
\end{gathered}
$$




$$
\begin{gathered}
\mathrm{p}\left(\mathrm{N}_{\mathrm{j}}\right)\left(\left(\phi_{11}+\left(\mathrm{N}_{\mathrm{j}}-2\right) \phi_{\mathrm{CC}}+\phi_{\mathrm{NN}}\right) \mathrm{W}+2 \sum_{\mathrm{n}=1}^{\mathrm{N}_{\mathrm{j}}-2}\left(\phi_{1 \mathrm{C}}+\left(\mathrm{N}_{\mathrm{j}}-2-\mathrm{n}\right) \phi_{\mathrm{CC}}+\phi_{\mathrm{CN}}\right) \mathrm{WQ}^{\mathrm{n}}+2 \phi_{1 \mathrm{~N}} \mathrm{WQ}^{\mathrm{N}_{\mathrm{j}}-1}\right) \\
+\ldots \\
\left.\left.+\mathrm{p}\left(\mathrm{N}_{\max }\right)\left(\left(\phi_{11}+\left(\mathrm{N}_{\max }-2\right) \phi_{\mathrm{CC}}+\phi_{\mathrm{NN}}\right) \mathrm{W}+2 \sum_{\mathrm{n}=1}^{\mathrm{N}_{\max }-2}\left(\phi_{1 \mathrm{C}}+\left(\mathrm{N}_{\max }-2-\mathrm{n}\right) \phi_{\mathrm{CC}}+\phi_{\mathrm{CN}}\right) \mathrm{WQ}^{\mathrm{n}}+2 \phi_{1 \mathrm{~N}} \mathrm{WQ}^{\mathrm{N}_{\max }-1}\right)\right)\right)
\end{gathered}
$$

All terms can be grouped together as a function of the exponent of Q (i.e. as a function of $n)$ :

$$
\begin{aligned}
& \mathrm{n}=0 \text { term, } \quad\left(\phi_{11^{\prime}}+\left(\phi_{11}+\phi_{\mathrm{NN}}\right) \sum_{\mathrm{N}_{\mathrm{j}}=2}^{\mathrm{N}_{\max }} \mathrm{p}\left(\mathrm{N}_{\mathrm{j}}\right)+\phi_{\mathrm{CC}} \sum_{\mathrm{N}_{\mathrm{j}}=3}^{\mathrm{N}_{\max }} \mathrm{p}\left(\mathrm{N}_{\mathrm{j}}\right)\left(\mathrm{N}_{\mathrm{j}}-2\right)\right) \mathrm{WQ}^{0} \\
& \mathrm{n}=1, \quad 2\left(\mathrm{p}(2) \phi_{1 \mathrm{~N}}+\left(\phi_{1 \mathrm{C}}+\phi_{\mathrm{CN}}\right) \sum_{\mathrm{N}_{\mathrm{j}}=3}^{\mathrm{N}_{\max }} \mathrm{p}\left(\mathrm{N}_{\mathrm{j}}\right)+\phi_{\mathrm{CC}} \sum_{\mathrm{N}_{\mathrm{j}}=4}^{\mathrm{N}_{\max }} \mathrm{p}\left(\mathrm{N}_{\mathrm{j}}\right)\left(\mathrm{N}_{\mathrm{j}}-3\right)\right) \mathrm{WQ}^{1} \\
& \mathrm{n}=2, \quad 2\left(\mathrm{p}(3) \phi_{1 \mathrm{~N}}+\left(\phi_{1 \mathrm{C}}+\phi_{\mathrm{CN}}\right) \sum_{\mathrm{N}_{\mathrm{j}}=4}^{\mathrm{N}_{\max }} \mathrm{p}\left(\mathrm{N}_{\mathrm{j}}\right)+\phi_{\mathrm{CC}} \sum_{\mathrm{N}_{\mathrm{j}}=5}^{\mathrm{N}_{\max }} \mathrm{p}\left(\mathrm{N}_{\mathrm{j}}\right)\left(\mathrm{N}_{\mathrm{j}}-4\right)\right) \mathrm{WQ}^{2}
\end{aligned}
$$

This can be defined recursively for the first $\mathrm{N}_{\max }-3$ terms as:

$$
1 \leq \mathrm{n} \leq \mathrm{N}_{\max }-3
$$

$2\left(\mathrm{p}(\mathrm{n}+1) \phi_{1 \mathrm{~N}}+\left(\phi_{1 \mathrm{C}}+\phi_{\mathrm{CN}}\right) \sum_{\mathrm{N}_{\mathrm{j}}=\mathrm{n}+2}^{\mathrm{N}_{\max }} \mathrm{p}\left(\mathrm{N}_{\mathrm{j}}\right)+\phi_{\mathrm{CC}} \sum_{\mathrm{N}_{\mathrm{j}}=\mathrm{n}+3}^{\mathrm{N}_{\max }} \mathrm{p}\left(\mathrm{N}_{\mathrm{j}}\right)\left(\mathrm{N}_{\mathrm{j}}-\mathrm{n}-2\right)\right) \mathrm{WQ}^{\mathrm{n}}$,

In addition, we have two specific expressions:

$$
\begin{aligned}
& \mathrm{n}=\mathrm{N}_{\max }-2, \quad 2\left(\mathrm{p}\left(\mathrm{N}_{\max }-1\right) \phi_{1 \mathrm{~N}}+\left(\phi_{1 \mathrm{C}}+\phi_{\mathrm{CC}}\right) \mathrm{p}\left(\mathrm{N}_{\max }\right)\right) \mathrm{WQ}^{\mathrm{N}_{\text {max }}-2} \\
& \mathrm{n}=\mathrm{N}_{\text {max }-1}, \quad 2\left(\mathrm{p}\left(\mathrm{N}_{\text {max }}\right) \phi_{1 \mathrm{~N}}\right) \mathrm{WQ}^{\mathrm{N}_{\text {max }}-1}
\end{aligned}
$$

So, for $\mathrm{N}_{\max }>2$

$$
\begin{gathered}
\overline{\operatorname{Int}}(\overrightarrow{\mathrm{s}})=\operatorname{Spur}\left(\operatorname { R e } \left(\left(\mathrm{p}(1) \phi_{1^{\prime} 1^{\prime}}+\left(\phi_{11}+\phi_{\mathrm{NN}}\right) \sum_{\mathrm{N}_{\mathrm{j}}=2}^{\mathrm{N}_{\text {max }}} \mathrm{p}\left(\mathrm{N}_{\mathrm{j}}\right)+\sum_{\mathrm{N}_{\mathrm{j}}=3}^{\mathrm{N}_{\text {max }}} \mathrm{p}\left(\mathrm{N}_{\mathrm{j}}\right)\left(\mathrm{N}_{\mathrm{j}}-2\right) \phi_{\mathrm{CC}}\right) \mathrm{W}\right.\right. \\
+2 \sum_{\mathrm{n}=1}^{\mathrm{N}_{\text {max }}-2}\left(\mathrm{p}(\mathrm{n}+1) \phi_{1 \mathrm{~N}}+\left(\phi_{1 \mathrm{C}}+\phi_{\mathrm{CN}}\right) \sum_{\mathrm{N}_{\mathrm{j}}=\mathrm{n}+2}^{\mathrm{N}_{\max }} \mathrm{p}\left(\mathrm{N}_{\mathrm{j}}\right)+\phi_{\mathrm{CC}} \sum_{\mathrm{N}_{\mathrm{j}}=\mathrm{n}+3}^{\mathrm{N}_{\max }} \mathrm{p}\left(\mathrm{N}_{\mathrm{j}}\right)\left(\mathrm{N}_{\mathrm{j}}-\mathrm{n}-2\right)\right) \mathrm{WQ}^{\mathrm{n}} \\
\left.\left.+2 \mathrm{p}\left(\mathrm{N}_{\max }\right) \phi_{1 \mathrm{~N}} \mathrm{WQ}^{\mathrm{N}_{\text {max }}-1}\right)\right)
\end{gathered}
$$


or

$$
\begin{gathered}
\overline{\operatorname{Int}}(\overrightarrow{\mathrm{s}})=\operatorname{Spur}\left(\operatorname { R e } \left(\left(\mathrm{C}_{1} \phi_{1^{\prime} 1^{\prime}}+\mathrm{C}_{2}\left(\phi_{11}+\phi_{\mathrm{NN}}\right)+\mathrm{C}_{3} \phi_{\mathrm{CC}}\right) \mathrm{W}\right.\right. \\
+\sum_{\mathrm{n}=1}^{\mathrm{N}_{\max }-2}\left(\mathrm{~K}_{1}(\mathrm{n}) \phi_{1 \mathrm{~N}}+\mathrm{K}_{2}(\mathrm{n})\left(\phi_{1 \mathrm{C}}+\phi_{\mathrm{CN}}\right)+\mathrm{K}_{3}(\mathrm{n}) \phi_{\mathrm{CC}}\right) \mathrm{WQ}^{\mathrm{n}} \\
\left.\left.+\mathrm{C}_{4} \phi_{1 \mathrm{~N}} \mathrm{WQ}^{\mathrm{N}_{\max }-1}\right)\right)
\end{gathered}
$$

with $\mathrm{C}_{1}=\mathrm{p}(1), \mathrm{C}_{2}=\sum_{\mathrm{N}_{\mathrm{j}}=2}^{\mathrm{N}_{\max }} \mathrm{p}\left(\mathrm{N}_{\mathrm{j}}\right), \mathrm{C}_{3}=\sum_{\mathrm{N}_{\mathrm{j}}=3}^{\mathrm{N}_{\max }} \mathrm{p}\left(\mathrm{N}_{\mathrm{j}}\right)\left(\mathrm{N}_{\mathrm{j}}-2\right), \mathrm{C}_{4}=2 \mathrm{p}\left(\mathrm{N}_{\max }\right), \mathrm{K}_{1}(\mathrm{n})=2 \mathrm{p}(\mathrm{n}+1)$,

$$
\mathrm{K}_{2}(\mathrm{n})=2 \sum_{\mathrm{N}_{\mathrm{j}}=\mathrm{n}+2}^{\mathrm{N}_{\max }} \mathrm{p}\left(\mathrm{N}_{\mathrm{j}}\right), \mathrm{K}_{3}(\mathrm{n})=2 \sum_{\mathrm{N}_{\mathrm{j}}=\mathrm{n}+3}^{\mathrm{N}_{\max }} \mathrm{p}\left(\mathrm{N}_{\mathrm{j}}\right)\left(\mathrm{N}_{\mathrm{j}}-\mathrm{n}-2\right)
$$

\section{RESULTS}

Calculation of XRD patterns for hypothetical structure models having different outer surface layers of the crystals

The formalism described above was used to implement an algorithm for the calculation of XRD patterns containing only $00 \ell$ basal reflections. Corrections for the Lorentz-polarization factor and instrumental variables such as horizontal and vertical beam divergences, goniometer radius, and dimension and thickness of samples have been introduced according to the recommendation of Reynolds (1986) and Drits et al. (1993). These corrections allow the simulation of XRD patterns that can be directly compared with experimental ones (Drits et al., 1997a; Sakharov et al., 1999; Lindgreen et al., 2000; Claret et al., 2002, 2004).

XRD patterns showing the influence of different OSLs were calculated for chlorite and mixed-layer chlorite-smectite (Ch-S) structure models. Chlorite is usually a trioctahedral mineral and the idealized composition for its 2:1 and 0:1 layers may be represented as $\left(\mathrm{Si}_{4-}\right.$ $\left.{ }_{x} \mathrm{Al}_{\mathrm{x}}\right)\left(\mathrm{Mg}, \mathrm{Fe}^{2+}\right)_{3} \mathrm{O}_{10}(\mathrm{OH})_{2}$ and $\left(\mathrm{Mg}, \mathrm{Fe}^{2+}\right)_{3-\mathrm{x}} \mathrm{Al}_{\mathrm{x}}(\mathrm{OH})_{6}$, respectively. $\mathrm{z}$-coordinates of the constituting atoms were taken from Moore and Reynolds (1989). Three chlorite models which differ from each other by their OSLs were considered. In the first two models chlorite crystals 
are terminated by 2:1 and 0:1 layers, respectively, whereas the third model corresponds to a periodic chlorite structure in which each crystal is terminated by a 2:1 layer on the one side and by a 0:1 layer on the other. XRD patterns calculated for such (Fe-free) chlorite models are shown on Figure 1. A dramatic redistribution of $00 \ell$ reflections intensities is observed when only one outer $0: 1$ layer is added to the $2: 1$ surface layer of the periodic chlorite crystals. In particular, relative intensity of 001 reflection is strongly increased or decreased when both surface layers of the chlorite crystals are represented by $2: 1$ or $0: 1$ layers, respectively. XRD patterns calculated for different cation compositions of 2:1 and 0:1 layers demonstrate the same effect. For each given composition and mean thickness of chlorite crystals the intensity of the 001 reflection significantly decreases when OSLs are 0:1 layers, even if the mean and maximum numbers of chlorite layers are high. Similarly, a strong increase of the 001 reflection intensity is observed when 2:1 layers are present on the outer surface whatever the chlorite composition.

In a chlorite-smectite (Ch-S) mixed-layer clay, 2:1 layers are separated from each other either by brucite-like sheets (chlorite interlayers) or by exchangeable cations and water or ethylene glycol (EG) molecules (smectite interlayers). Structure models were constructed using the parameters given by Moore and Reynolds (1989). In addition, three different types of OSLs were considered for Ch-S crystals when both their sides are terminated by 2:1 layers, by smectite interlayers and by brucite-like sheets. Ch-S structures terminated by $2: 1$ layers correspond to Reynolds model of Ch-S. XRD patterns were calculated for Ch-S core models in which $60 \%$ of chlorite and $40 \%$ of smectite interlayers are interstratified at random. Figure 2 shows that only the reflection near $15-16 \AA$ is sensitive to the OSL nature in Ch-S crystals. Its intensity significantly decreases for the model in which both OSLs are 0:1 layers. This is true whatever the amount and the distribution of Fe atoms in the 2:1 and 0:1 layers. Figure 2 shows two other varieties along with Fe-free $\mathrm{Ch}-\mathrm{S}$ (top part of figure 2). In the first one, Fe atoms are 
located only in the 2:1 layers (middle part of figure 2) and only in 0:1 sheets in the other model (bottom part of figure 2).

Analysis of XRD patterns calculated for Ch-S differing by the content and cation composition of their interstratified layers has revealed the following features: (1) the influence of different OSLs is significant even for relatively high smectite content (40\%), but decreases with increasing smectite content; (2) the nature of OSLs modifies mostly the intensity of the first low-angle basal reflections whereas the relative intensities of the other reflections are mostly unaffected by the terminating layer type, independently on the layer type, cation composition and distribution; (3) for a given $\mathrm{Ch}: \mathrm{S}$ ratio the intensity of the first basal reflection decreases for Ch-S crystals terminated by a 0:1 layer, whereas more subtle differences are observed between XRD patterns calculated for Ch-S crystals that end on 2:1 layers or smectite interlayers; (4) different terminating layers change not only the intensity but also the position of the first basal reflection.

On the other hand, XRD patterns calculated for EG-solvated kaolinite-smectite MLSs (K-S) differing by their OSLs are almost identical for a given composition, whatever the nature of the OSL (kaolinite or smectite layer - data not shown). Likewise, similar XRD patterns are calculated for EG-solvated illite-smectite MLSs (I-S) with either 2:1 layers on both sides or with a 2:1 layer on one side and either smectite or illite layer (depending on the I-S composition) on the other. When I-S crystals are terminated on both sides by one sheet of EG molecules significant modifications of position and profile are observed for the second order basal reflection at 8.5-10.0 $\AA$ (data not shown). However, according to our experience, similar diffraction effects may be result from the modification of other structural, chemical and probability parameters describing such I-S structures, and it is not clear if the nature of OSLs can be determined from XRD modelling for I-S MLSs. 


\section{Simulation of the experimental XRD patterns}

The actual structure of OSLs was studied for two monomineralic chlorites and one regular Ch-S (corrensite). The two chlorite samples have different contents of octahedral Fe cations and their structural formulae are given in Table 1. For structure models of chlorite zcoordinates of atoms have been derived from the single-crystal refinements of chlorite structures with cation compositions close to those of the studied samples (Drits and SmoliarZvyagina, 1992). XRD patterns were calculated for chlorite models having the three possible combinations of layer terminations mentioned above. For each model the number of layers in crystals was described by a log-normal distribution with mean and maximum $\mathrm{N}$ equal to 30 and 150 layers (Drits et al., 1997b). Figures 3 and 4 show that for both samples the best fit to the experimental intensity distribution is obtained when crystals are terminated by brucite layers on both ends. For the other two models a significant disagreement between experimental and calculated intensities of $00 \ell$ reflections is observed (Figures $3 b, c$, and $4 b, c)$.

Similar calculations were carried out for a corrensite sample in both air-dried and EGsolvated states. Corrensite structural formula is given in Table 1 and z-coordinates of atoms for smectite and chlorite layers were taken from Moore and Reynolds (1989). XRD patterns were calculated for structure models having different OSLs: brucite-like sheets; 2:1 layers or smectite interlayers. Comparison of experimental and calculated XRD patterns shows that for both EG-solvated and air-dried states the best fit is obtained when corrensite crystals are terminated by smectite interlayers (Figures 5,6). Thus, the nature of crystal outer surfaces depends on a mineral structure and can reflect physico-chemical conditions of its formation. For the studied chlorite crystals it is represented by $(\mathrm{OH})$ groups and for corrensite crystals by exchangeable cations and water molecules. 


\section{DISCUSSION}

For irregular MLSs the influence of OSLs on diffraction intensities cannot be predicted because it is not possible to consider separately the contributions to diffraction of the interference function and of the scattering power for different layer types in such structures. In contrast, the influence of OSL nature on diffracted intensity can be estimated for structures containing only one L-type layer. One can show that an intensity distribution along the $\mathrm{c}^{*}$ axis for a microcrystal consisting of $\mathrm{N}$ identical layers and ending on one side by $\mathrm{L}_{l}$ and on the other side by $\mathrm{L}_{u}$ layers can be expressed as:

$$
\begin{gathered}
I\left(z^{*}\right)=F F^{*}\left[N+2 \operatorname{Re} \sum_{n=1}^{N-1}(N-n) \exp \left(-2 \pi i n z^{*} d_{001}\right)\right]+ \\
F_{1} F_{1}^{*}+F_{u} F_{u}^{*}+2 \operatorname{Re}\left(F_{1}^{*} F+F^{*} F_{u}\right) \sum_{n=1}^{N-1} \exp \left(-2 \pi i n z^{*} d_{001}\right)+ \\
2 \operatorname{ReF}_{1}^{*} F_{u} \exp \left(-2 \pi i(N-1) z^{*} d_{001}\right)
\end{gathered}
$$

where $\mathrm{F}, \mathrm{F}_{l}$ and $\mathrm{F}_{u}$ are the structure factors of $\mathrm{L}, \mathrm{L}_{l}$ and $\mathrm{L}_{u}$ layers, $\mathrm{z}^{*}$ is the coordinate along the $\mathrm{c}^{*}$ axis, and $\mathrm{d}_{001}$ is the periodicity of the core crystal along the $\mathrm{c}^{*}$ axis. When $\mathrm{z}^{*} \mathrm{~d}_{001}=l$ this intensity may be expressed as:

$$
\mathrm{I}=\mathrm{N}^{2} \mathrm{~F}^{2}+\mathrm{F}_{l}^{2}+\mathrm{F}_{u}{ }^{2}+2 \mathrm{NRe}\left(\mathrm{F}_{l}^{*} \mathrm{~F}+\mathrm{F}^{*} \mathrm{~F}_{u}\right)+2 \operatorname{Re}\left(\mathrm{F}_{l}^{*} \mathrm{~F}_{u}\right)
$$

In this formula numerical values of each term can be calculated separately for each given $z^{*}$ if structures of the core layers and of each OSL are known. For example, Tables 2 and 3 contain these values calculated for chlorite crystals differing from each other by their OSLs. It is remarkable that the values reflecting the interaction of an outer brucite-like layer with core layers of the core crystal in all layer subsequences in which the first one is the outer brucite-like layer $\left(2 \mathrm{NRe}\left(\mathrm{F}_{b}{ }^{*} \mathrm{~F}\right)-4^{\text {th }}\right.$ column, Table 2$)$ are compatible with those corresponding to the core layers $\left(\mathrm{NF}^{2}(00 \ell)-2^{\text {nd }}\right.$ column, Table 2$)$. Moreover, the former values may be positive or negative for different $\ell$ values. These specific features explain why adding only one brucite-like sheet to the core chlorite crystals ( 5 layers $-70 \AA$ ) dramatically modifies the intensity 
distribution of basal reflections when both sides of the chlorite crystals are terminated by brucite-like layers (see the $2^{\text {nd }}$ and last two columns in Table 2).

In Table 3, similar calculations are reported for chlorite crystals (four 2:1:1 layers in the core crystal) with 2:1 layers as OSLs. Despite the low values of $\mathrm{F}_{t}^{2}$ corresponding to the 2:1 layer, the interaction of such OSLs with core layers contributes significantly to the diffracted intensity $\left(2 \mathrm{NRe}\left(\mathrm{F}_{t}{ }^{*} \mathrm{~F}\right)-4^{\text {th }}\right.$ column, Table 3$)$. This is especially so for the first two reflections as the term $2 \mathrm{NRe}\left(\mathrm{F}^{*} \mathrm{~F}_{t}\right)$ is respectively positive and negative signs for the first and second order reflections. As a consequence chlorite crystals terminated on both sides by 2:1 layers have stronger 001 reflection than purely periodic chlorite crystals with one $2: 1$ layer on one side and a brucite layer on the other side.

Equation (39) can be used in a similar way to calculate diffraction effects for any periodic layer structure whatever their OSLs. For example, the 001 reflection calculated for 10layer thick periodic kaolinite crystals is $\sim 30 \%$ more intense than that calculated for the same kaolinite crystals assuming a pyrophillite-like outer layer forming hydrogen bonds with the last kaolinite core layer.

It is also clear from equation (39) that the influence of OSLs on the calculated XRD patterns decreases with the increasing number of core layers. However, this influence depends on the structure of both core and OS layers and significant modifications of the basal-reflection intensity distribution may be observed even for 30-layer thick ( $>420 \AA)$ chlorite crystals (Figures 3, 4).

Finally, equation (39) shows that the main influence on the diffracted intensity distribution arises from the interaction of OSLs with crystal core layers. The effect resulting from these interactions strongly depends on the structural amplitudes of both core and OS layers and may be significant even when the scattering power of OSLs is negligible. For periodic structures the diffraction effects resulting from the presence of different OSLs may be predicted whereas such a prediction is not possible for MLSs even though similar interactions 
are involved. Preliminary calculations have shown that among the three main groups of natural mixed-layer clays (I-S, Ch-S, and K-S) the influence of OSLs on XRD patterns is significant only for MLSs containing chlorite layers. For other MLSs, additional hindrance to the characterization of OSL nature by XRD is related to the influence of chemical and structural parameters on XRD patterns, as diffraction effects similar to those resulting from the presence of different OSLs can be obtained, for example, by varying the layer chemistry and/or stacking sequence.

\section{CONCLUSION}

The theoretical approach described in the present work provides the opportunity to determine the nature of OSLs in MLS from the simulation of XRD patterns. With this respect the results obtained in the present study for the chlorite samples are remarkable as they demonstrate that relative intensities of the odd reflections depend not only on the distribution of Fe in the chlorite structure over the 2:1 and 0:1 layers, but also on the nature of these OSLs.

For periodic structures containing only one layer type, the influence OSLs may be predicted from simple calculations, and is independent of the scattering power of the OSL. Such a prediction is not possible for MLSs. In addition, comparison of the OSL nature determined from XRD profile modeling with that deduced from direct observations using electron or atomic force microscopies (e.g.) is crucial for MLSs because of the similar diffraction effects that may be obtained by varying structural and chemical parameters of the MLS on the one hand and the OSL nature on the other. Among the usual MLSs found in natural samples, most significant effects have been calculated for those containing elementary chlorite layers.

The knowledge of the OSL nature may be used to understand better surface properties of MLSs, and more especially of layer silicates, and/or to derive constrains on their growth 
conditions. Accordingly, the systematic presence of 0:1 layers on the two sides of crystals in the reported chlorite structures may reflect specific growth conditions of these chlorites.

\section{ACKNOWLEDGMENTS}

The authors thank S. Hillier for chlorite samples and D.K. McCarty for recording XRD patterns and for improving the English and the clarity of the manuscript. BAS is grateful to the University of Orléans for financial support. BAS and VAD thank the Russian Science Foundation for partial financial support. BL acknowledges financial support from the CNRS/PICS 709 program. AE R.T. Cygan, R.C. Reynolds, and an anonymous reviewer are thanked for their helpful suggestions.

\section{REFERENCES}

Claret, F., Bauer, A., Schafer, T., Griffault, L. and Lanson, B. (2002) Experimental investigation of the interaction of clays with high-pH solutions: A case study from the Callovo-Oxfordian formation, Meuse-Haute Marne underground laboratory (France). Clays and Clay Minerals, 50, 633-646.

Claret F., Sakharov B.A., Drits V.A., Velde B., Meunier A., Griffault L. and Lanson B. (2004) Clay minerals in the Meuse - Haute Marne underground laboratory (France): Possible influence of organic matter on clay mineral evolution? Clays \& Clay Minerals, in press.

Drits, V.A. and Sakharov B.A. (1976) X-ray structure analysis of interstratified minerals. Nauka, Moscow, 256pp. (in Russian).

Drits, V.A. and Smoliar-Zvyagina, B.B. (1992) Relations between unit-cell parameters and cation composition of sheet silicates. II. Trioctahedral chlorites. Geologica CarpathicaClays, 1, 35-40. 
Drits, V.A. and Tchoubar, C. (1990) X-ray diffraction by disordered lamellar structures. Springer Verlag, Berlin, 371pp.

Drits, V.A., Kameneva, M.Yu., Sakharov, B.A., Dainyak, L.G., Tsipursky, S.I., SmoliarZvyagina, B.B., Bookin, A.S. and Salyn, A.L. (1993) Problems of determination of the actual structure of glauconites and related fine-dispersed minerals. Nauka, Novosibirsk, 200pp. (in Russian).

Drits, V.A., Sakharov, B.A., Lindgreen, H. and Salyn, A.L. (1997a) Sequential structure transformation of illite-smectite-vermiculite during diagenesis of Upper Jurassic shales from the North Sea and Denmark. Clay Minerals, 32, 351-371.

Drits, V.A., Środoń, J. and Eberl, D.D. (1997b) XRD measurement of mean crystallite thickness of illite and illite/smectite: Reappraisal of the Kubler index and the Scherrer equation. Clays and Clay Minerals, 45, 461-475.

Kakinoki, J. and Komura, Y. (1952) Intensity of X-ray diffraction by one dimensionally disordered crystals. I: General derivation in the case of the "Reichweite" $\mathrm{S}=0$ and 1. Journal of the Physical Society of Japan, 7, 30-35.

Kakinoki, J. and Komura, Y. (1954a) Intensity of X-ray diffraction by one-dimensionally disordered crystals. The close packed structure. Journal of the Physical Society of Japan, 9, 177-183.

Kakinoki, J. and Komura, Y. (1954b) Intensity of X-ray diffraction by one dimensionally disordered crystals. II: General derivation in the case of the correlation range $\mathrm{S} \geq 2$. Journal of the Physical Society of Japan, 9, 169-176.

Kakinoki, J. and Komura, Y. (1965) Diffraction by a one-dimensionally disordered crystal. I: The intensity equation. Acta Crystallographica, 19, 137-147.

Lindgreen, H., Drits, V.A., Sakharov, B.A., Salyn, A.L., Wrang, P. and Dainyak, L.G. (2000) Illite-smectite structural changes during metamorphism in black Cambrian Alum shales from the Baltic area. American Mineralogist, 85, 1223-1238. 
Ma, C. and Eggleton, R.A. (1999) Surface layer types of kaolinite: high resolution transmission electron microscopy study. Clays and Clay Minerals, 47, 181-191.

Moore, D.M. and Reynolds, R.C. (1989) X-ray diffraction and the identification and analysis of clay minerals. Oxford University Press, Oxford, 332 pp.

Plançon A. (1981) Diffraction by layer structures containing different kinds of layers and stacking faults. Journal of Applied Crystallography, 14, 300-304.

Plançon A. (2002) New modeling of X-ray diffraction by disordered lamellar structures, such as phyllosilicates. American Mineralogist, 87, 1672-1677.

Plançon A. (2003) Modelling X-ray diffraction by lamellar structures composed of electrically charged layers. Journal of Applied Crystallography, 36, 146-153.

Plançon, A. and Tchoubar, C. (1976) Etude des fautes d'empilement dans les kaolinites partiellement désordonnées. Journal of Applied Crystallography, 9, 279-285.

Reynolds R.C. (1967) Interstratified clay system: calculation of the total one-dimensional diffraction function. American Mineralogist, 52, 661-672.

Reynolds R.C. (1980) Interstratified clay minerals. Pp. 249-303 in: Crystal Structures of Clay Minerals and Their X-ray Identification (G.W. Brindley and G. Brown, editors). Mineralogical Society, London.

Reynolds R.C. (1986) The Lorentz factor and preferred orientation in oriented clay aggregates. Clays and Clay Minerals, 34, 359-367.

Sakharov, B.A., Naumov, A.S. and Drits, V.A. (1982a) X-ray diffraction by mixed-layer structures with random distribution of stacking faults. Doklady Akademii Nauk SSSR, 265, 339-343 (in Russian).

Sakharov, B.A., Naumov, A.S. and Drits, V.A. (1982b) X-ray intensities scattered by layer structure with short range ordering parameters $\mathrm{S} \geq 1$ and $\mathrm{G} \geq 1$. Doklady Akademii Nauk SSSR, 265, 871-874 (in Russian). 
Sakharov, B.A., Lindgreen, H., Salyn, A.L. and Drits, V.A. (1999) Determination of illitesmectite structures using multispecimen X-ray diffraction profile fitting. Clays and Clay Minerals, 47, 555-566.

Tsipursky, S.J., Eberl, D.D. and Buseck, P.R. (1992) Unusual tops (bottoms ?) of particles of 1M illite from the Silverton caldera (Co). Pp. 381-382 in: Proceedings of the American Society of Agronomy annual meeting, Minneapolis, 1992. American Society of Agronomy, Madison, WI. 


\section{FIGURE CAPTION}

Figure 1. XRD patterns calculated for Fe-free periodic chlorite crystals (b) and crystals terminated by 2:1 (a) and 0:1 (c) layers. XRD patterns were calculated using the following parameters: size of both Soller slits was $2.3^{\circ}$, length of the sample $-30 \mathrm{~mm}$, angular aperture of the divergence slit $-0.5^{\circ}$, goniometer radius $-175 \mathrm{~mm}$, orientation parameter $\sigma^{*}-12^{\circ}$, mean and maximum number of chlorite layers were 8 and 50 , respectively, for all calculations. Layer structure models were constructed using the parameters given by Moore and Reynolds (1989) and a layer thickness of $14.2 \AA$.

Figure 2. XRD patterns calculated for random mixed-layer chlorite-smectite models containing $60 \%$ of chlorite layers. The models differ from each other by amount and distribution of Fe in 2:1 and 0:1 layers. For each given content and distribution of Fe the upper, middle and lower XRD patterns correspond to Ch-S crystals terminated by 2:1 layers, smectite interlayers, or brucite sheets, respectively. Layer thickness for EG-solvated smectite layers was assumed to be $16.9 \AA$, whereas mean and maximum numbers of layers building up coherent scattering domains were 8 and 20, respectively, for all calculations. Other calculation parameters as in Figure 1.

Figure 3. Comparison of the experimental XRD pattern of chlorite 1 (see Table 1 for composition) with those calculated for chlorite crystals terminated by brucite sheets (a), one 2:1 layer and one brucite sheet (b) and 2:1 layers (c). Experimental and calculated XRD patterns are shown as crosses and solid line, respectively, whereas intensities of $00 \ell$ reflections are indicated by solid and dashed arrows for calculated and experimental patterns, respectively. Difference plots are shown below the compared XRD patterns. Experimental XRD patterns of these samples were recorded using $\mathrm{CuK} \alpha$ radiation with Scintag powder diffractometer equipped with Kevex Si (Li) solid detector. Two Soller slits $\left(2.5^{\circ}\right)$ and one divergence slit $\left(0.5^{\circ}\right)$ have been used. Intensity of $00 \ell$ reflections were 
measured for $30 \mathrm{sec}$. per $0.02^{\circ} 2 \theta$ step in the interval $1.5-35.0^{\circ} 2 \theta$. Length of samples was $35 \mathrm{~mm}$. Layer thickness for chlorite layers was $14.165 \AA$, whereas mean and maximum numbers of chlorite layers were 30 and 150, respectively. Other calculation parameters as in Figure 1.

Figure 4. Comparison of the experimental XRD pattern of chlorite 2 (see Table 1 for composition) with those calculated for chlorite crystals terminated by brucite sheets (a), one 2:1 layer and one brucite sheet (b) and 2:1 layers (c). Patterns and calculation parameters as in Figure 3, except for the chlorite layer thickness (14.190 $\AA$ ).

Figure 5. Comparison of the experimental XRD pattern of EG-solvated corrensite sample with those calculated for corrensite crystals terminated by smectite interlayers (a), brucite sheets (b) and 2:1 layers (c). Patterns as in Figure 3. Experimental XRD patterns were recorded using CoK $\alpha$ radiation with Philips PW3040 powder diffractometer equipped with a curved graphite diffracted-beam monochromator. Two Soller slits $\left(2.5^{\circ}\right)$, one divergence slit $\left(0.25^{\circ}\right)$ and one anti-scatter slit $\left(0.25^{\circ}\right)$ have been used. Intensity of $00 \ell$ reflections were measured for $10 \mathrm{sec}$. per $0.02^{\circ} 2 \theta$ step in the interval $1.5-35.0^{\circ} 2 \theta$. Length of samples was $35 \mathrm{~mm}$. Layer thickness for chlorite and EG-solvated smectite layers were $14.20 \AA$ and $16.80 \AA$, respectively, whereas mean and maximum number of layers building up coherent scattering domains were 12 and 70, respectively, for both calculations. A 50:50 smectite:chlorite ratio, and maximum possible degree of ordering $(\mathrm{R}=1)$ were assumed to describe layer stacking in corrensite. Other calculation parameters as for Figure 1.

Figure 6. Comparison of the experimental XRD pattern of corrensite sample in air-dried state with those calculated for corrensite crystals terminated by smectite interlayers (a), brucite sheets (b) and 2:1 layers (c). Patterns as in Figure 3. Layer thickness for AD smectite layers was $15.20 \AA \AA$. Other calculation parameters as in Figure 5. 
Table 1. Structural formulae of the two chlorite and of the corrensite samples.

\begin{tabular}{|c|c|c|c|c|c|c|c|c|c|}
\hline \multirow[t]{2}{*}{ Sample } & \multicolumn{5}{|c|}{ 2:1 layer } & \multicolumn{3}{|c|}{ 0:1 layer } & \multirow{2}{*}{$\begin{array}{c}\text { interlayer } \\
\mathrm{Ca}\end{array}$} \\
\hline & $\mathrm{Si}$ & $\mathrm{Al}^{\mathrm{IV}}$ & $\mathrm{Al}^{\mathrm{VI}}$ & $\mathrm{Fe}$ & $\mathrm{Mg}$ & $\mathrm{Al}^{\mathrm{VI}}$ & $\mathrm{Fe}$ & $\mathrm{Mg}$ & \\
\hline Chlorite 1 & 2.95 & 1.05 & - & 0.33 & 2.67 & 1.11 & 0.24 & 1.61 & - \\
\hline Chlorite 2 & 2.78 & 1.22 & - & 1.10 & 1.90 & 1.26 & 0.81 & 0.91 & - \\
\hline Corrensite & 6.80 & 1.20 & - & 0.44 & 5.56 & 0.80 & 0.24 & 1.96 & 0.20 \\
\hline $\begin{array}{r}\text { Note: } \\
\text { after dissolut }\end{array}$ & iical & & tions & & determ & ned $f$ & om we & $\begin{array}{l}\text { chem } \\
\mathrm{e}^{2+} \text { ar }\end{array}$ & $\begin{array}{l}\mathrm{Fe}^{3+} \\
\end{array}$ \\
\hline
\end{tabular}

Table 2. Respective contributions of core layers $\left(\mathrm{NF}^{2}\right)$, outer brucite layers $\left(\mathrm{F}_{b}{ }^{2}\right)$, and outer brucite layers interacting with core layers $\left(2 \mathrm{NReF}_{b}{ }^{*} \mathrm{~F}\right)$ to the diffracted intensity of $00 \ell$ reflections.

\begin{tabular}{cccccc}
\hline $00 \ell$ & $\mathrm{NF}^{2}$ & $\mathrm{~F}_{b}{ }^{2}$ & $2 \mathrm{NReF}_{b}{ }^{*} \mathrm{~F}$ & $\Sigma$ & $\Sigma$ \\
& $\left(\times 10^{3}\right)$ & $\left(\times 10^{3}\right)$ & $\left(\times 10^{3}\right)$ & & (norm.) \\
\hline 001 & 96 & 27 & -102 & 21 & 2 \\
002 & 440 & 16 & 169 & 625 & 45 \\
003 & 843 & 6.2 & 145 & 994 & 72 \\
004 & 1302 & 1.2 & 78 & 1381 & 100 \\
005 & 742 & 0.01 & -5.9 & 736 & 53 \\
\hline
\end{tabular}


Table 3. Respective contributions of core layers $\left(\mathrm{NF}^{2}\right)$ outer $2: 1$ layers $\left(\mathrm{F}_{t}^{2}\right)$, and outer 2:1 layers interacting with core layers $\left(2 \mathrm{NReF}_{t} \mathrm{~F}^{*}\right)$ to the diffracted intensity of $00 \ell$ reflections.

\begin{tabular}{cccccc}
\hline $00 \ell$ & $\mathrm{NF}^{2}$ & $\mathrm{~F}_{t}^{2}$ & $2 \mathrm{NReF}^{*} \mathrm{~F}_{t}$ & $\Sigma$ & $\Sigma$ \\
& $\left(\times 10^{3}\right)$ & $\left(\times 10^{3}\right)$ & $\left(\times 10^{3}\right)$ & & (norm.) \\
\hline 001 & 74 & 42 & 110 & 226 & 18 \\
002 & 337 & 1.9 & -51 & 288 & 24 \\
003 & 645 & 1.3 & 58 & 704 & 58 \\
004 & 996 & 12 & 216 & 1224 & 100 \\
005 & 568 & 12 & 168 & 748 & 61 \\
\hline
\end{tabular}




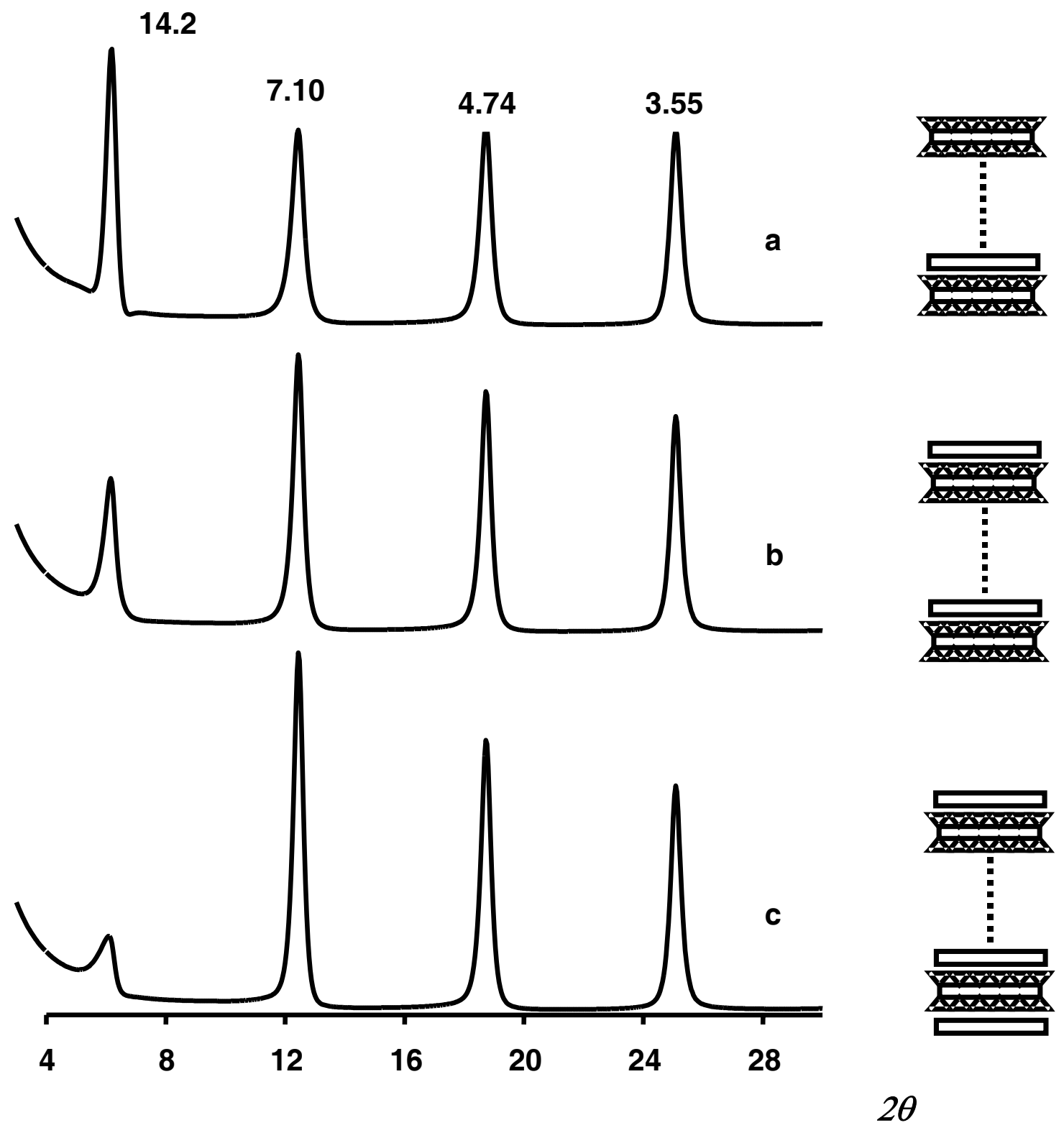

Sakharov et al., Fig. 1 


\section{Outer surface}

layers

2:1 layers

Smectite interlayer

Brucite
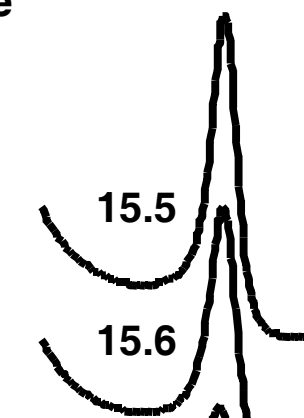

\subsection{3}

Fe - free
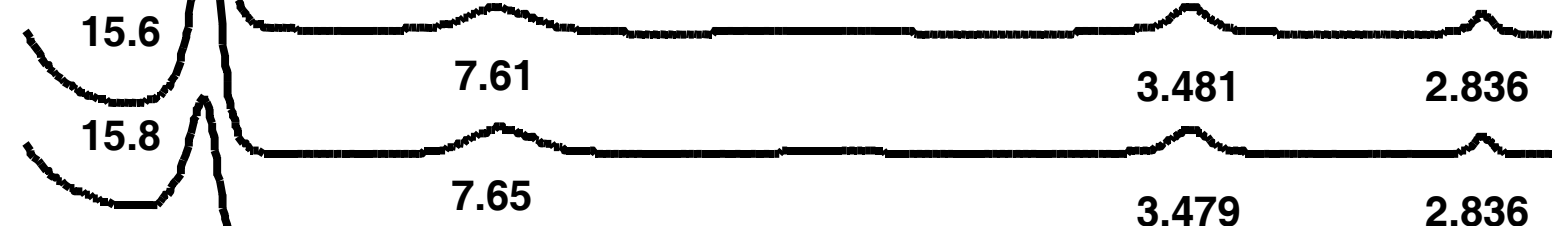

2:1 layers
Smectite
interlayer

Brucite

2:1 layers
Smectite
interlayer

Brucite

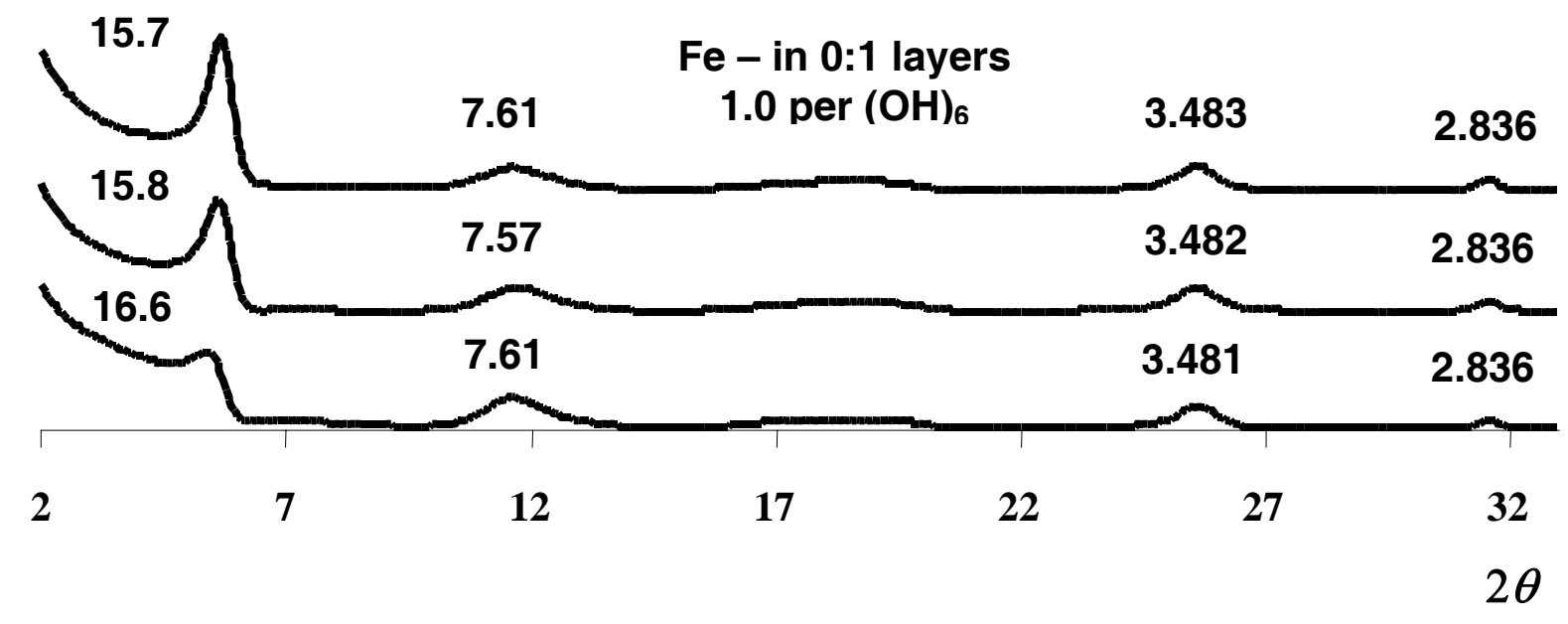




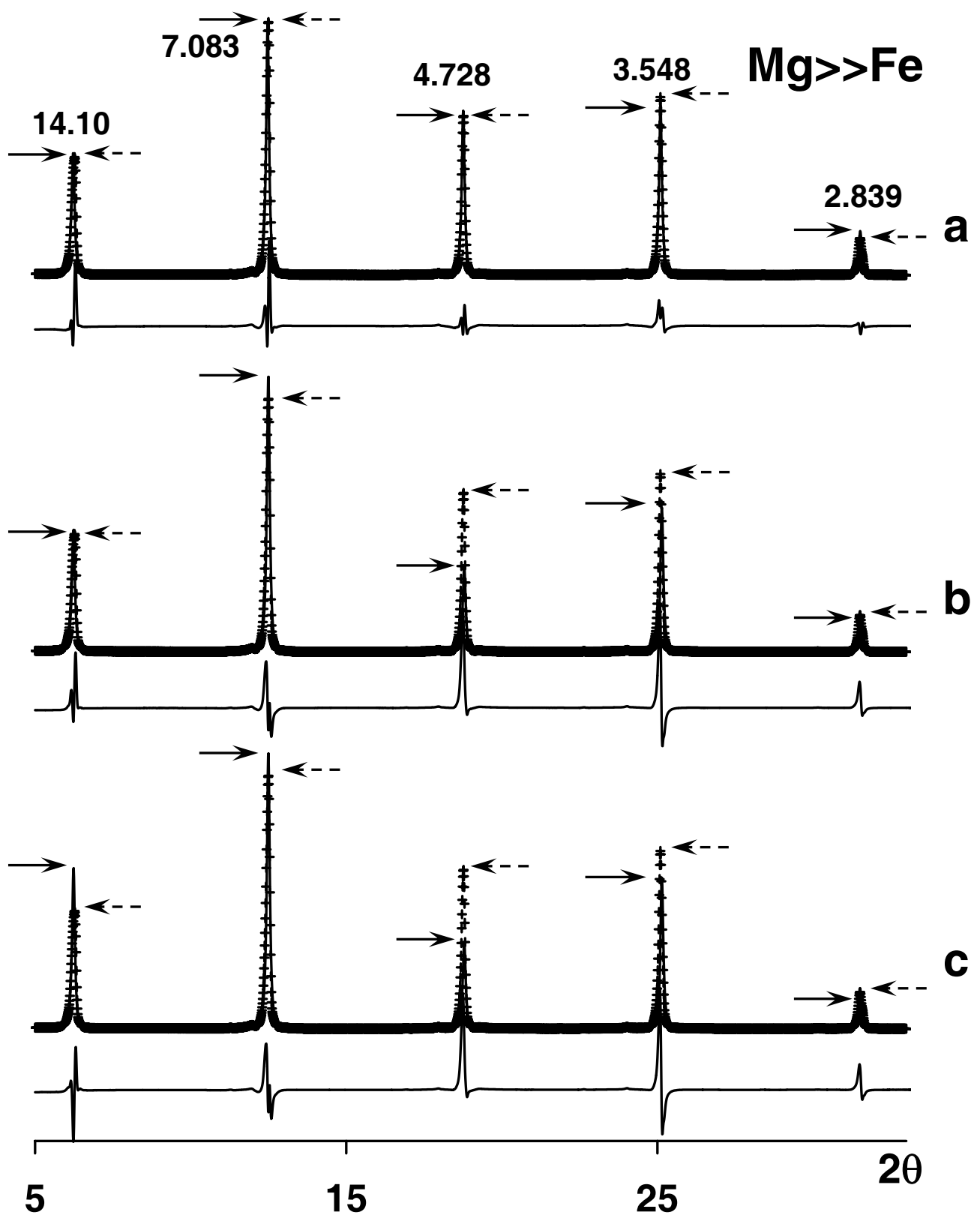

Sakharov et al., Fig. 3 


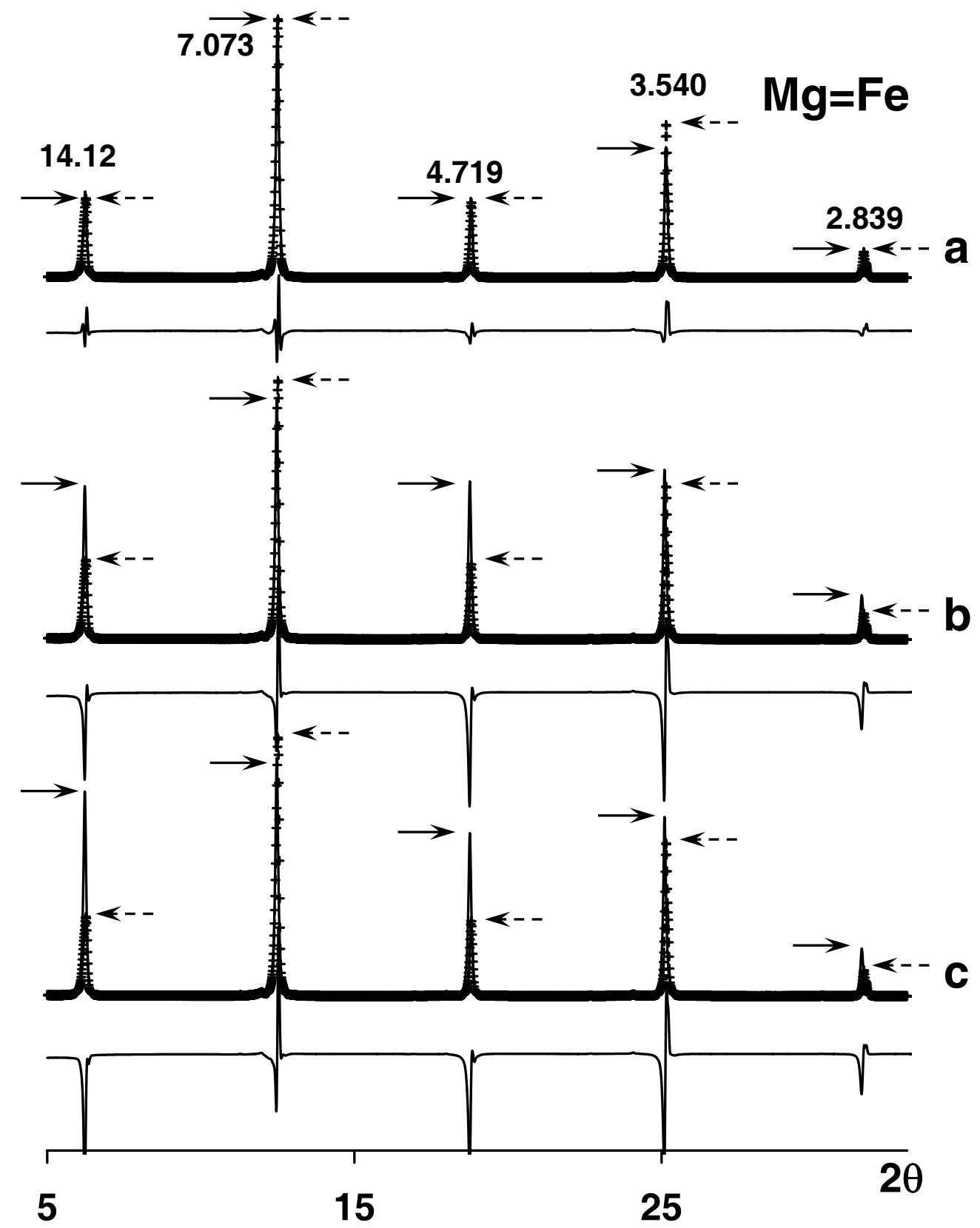

Sakharov et al., Fig. 4 


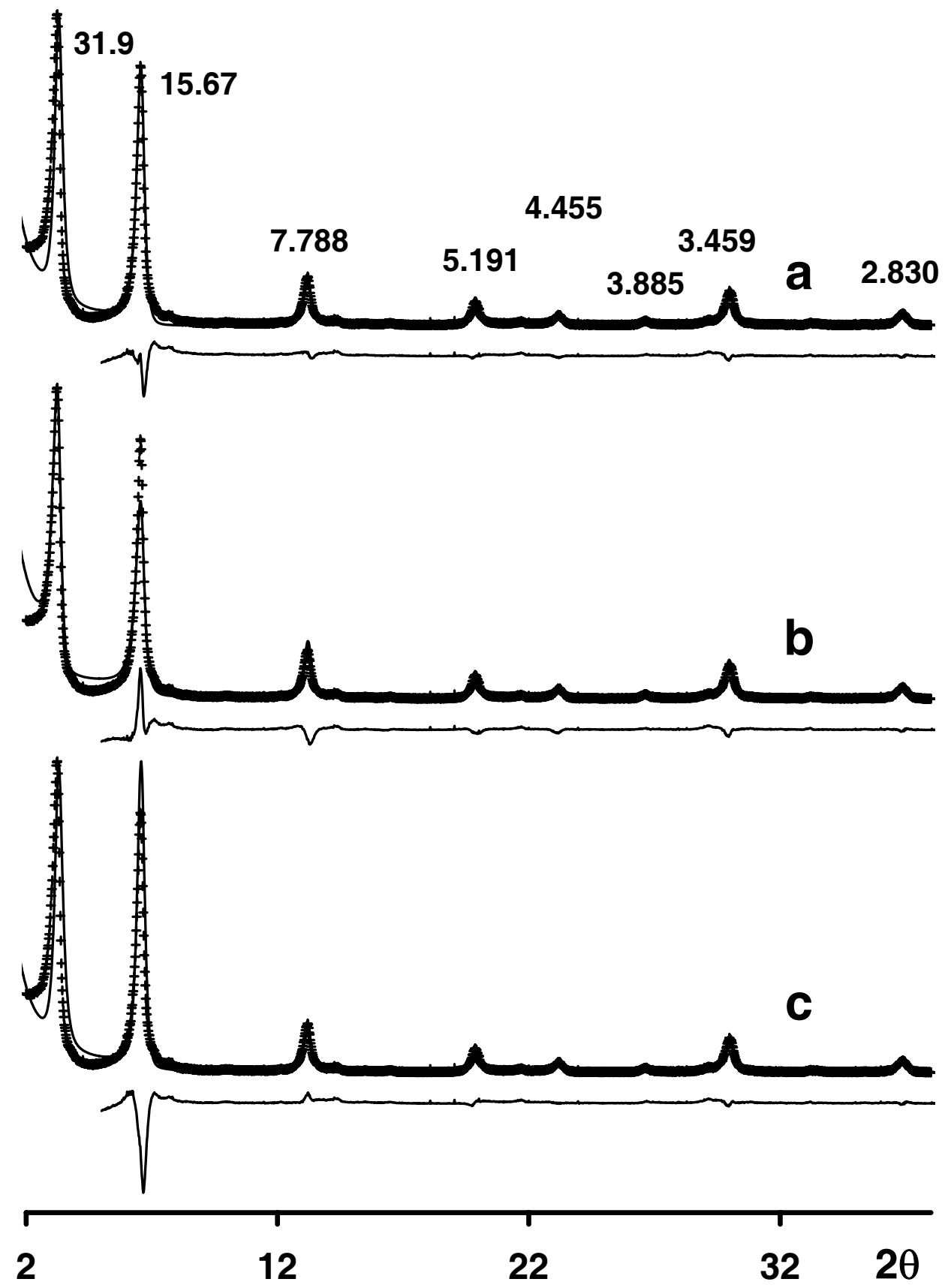

Sakharov et al., Fig. 5 


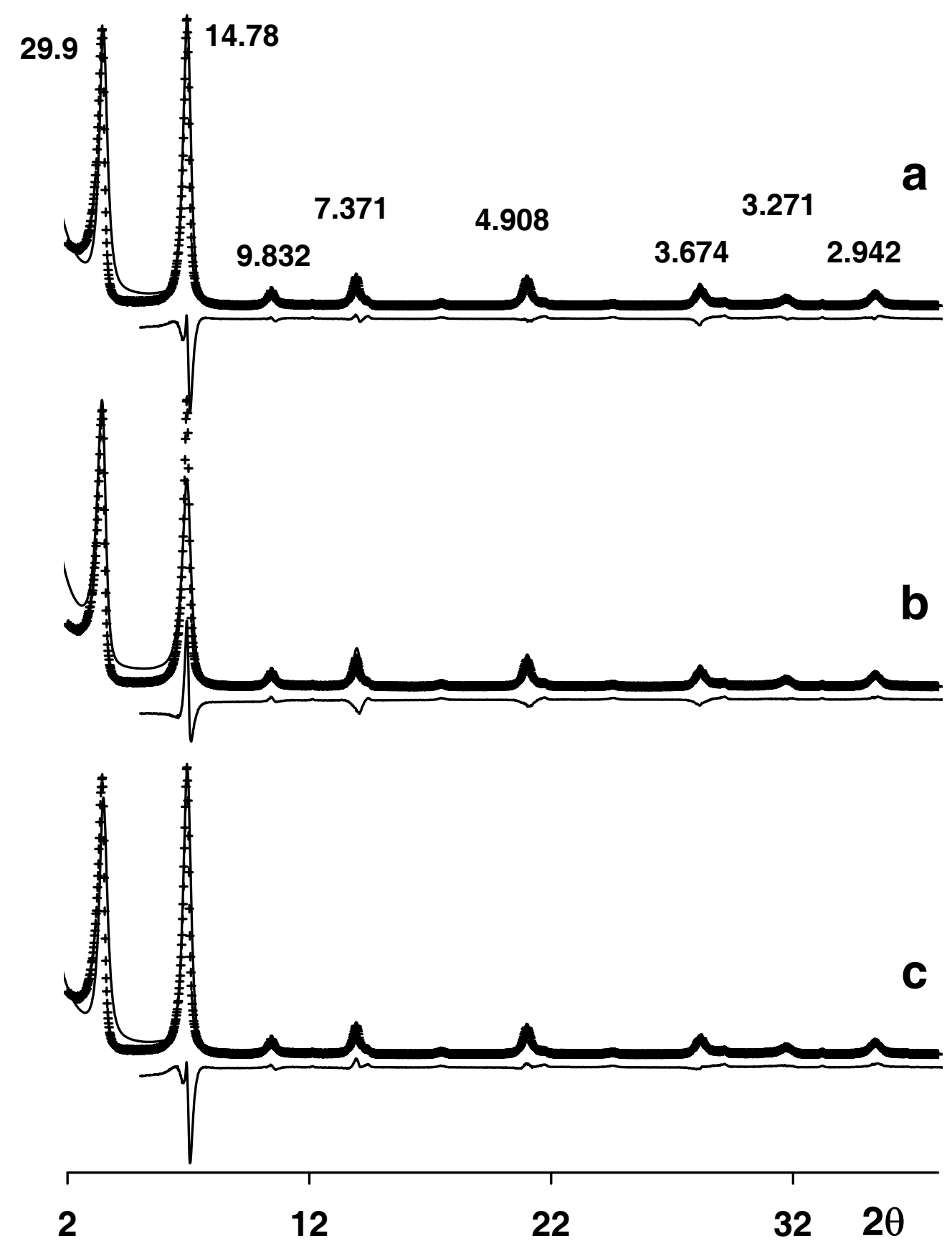

Sakharov et al., Fig. 6 